\title{
Observations of spatial and velocity structure in the Orion molecular cloud ${ }^{\star}, \star \star$
}

\author{
H. D. Nissen ${ }^{1}$, M. Gustafsson ${ }^{1}$, J. L. Lemaire ${ }^{2, \star \star \star}$, Y. Clénet $^{3}$, D. Rouan ${ }^{3}$, and D. Field ${ }^{1, \star \star \star}$ \\ 1 Department of Physics and Astronomy, University of Aarhus, 8000 Aarhus C, Denmark \\ e-mail: dfield@phys.au.dk \\ 2 Observatoire de Paris and Université de Cergy-Pontoise, LERMA and UMR 8112 du CNRS, 92195 Meudon, France \\ 3 Observatoire de Paris-Meudon, LESIA, 92195 Meudon Principal Cedex, France
}

Received 5 October 2005 / Accepted 15 December 2006

\begin{abstract}
Observations are reported of $\mathrm{H}_{2}$ IR emission in the $\mathrm{S}(1) v=1-0$ line at $2.121 \mu \mathrm{m}$ in the Orion Molecular Cloud, OMC1, using the GriF instrument on the Canada-France-Hawaii Telescope. GriF uses a combination of adaptive optics and Fabry-Perot interferometry, yielding a spatial resolution of $0.15^{\prime \prime}$ to $0.18^{\prime \prime}$ and velocity discrimination as high as $1 \mathrm{~km} \mathrm{~s}^{-1} .193$ bright $\mathrm{H}_{2}$ emission regions can be identified in OMC1. The general characteristics of these features are described in terms of radial velocities, brightness and spatial displacement of maxima of velocity and brightness, the latter to yield the orientation of flows in the plane of the sky. Strong spatial correlation between velocity and bright $\mathrm{H}_{2}$ emission is found and serves to identify many features as shocks. Important results are: (i) velocities of the excited gas illustrate the presence of a zone to the south of BN-IRc2 and Peak 1, and the west of Peak 2, where there is a powerful blue-shifted outflow with an average velocity of $-18 \mathrm{~km} \mathrm{~s}^{-1}$. This is shown to be the NIR counterpart of an outflow previously identified in the radio, originating from either source I or source n. (ii) There is a band of weak radial velocity features $\left(<5 \mathrm{~km} \mathrm{~s}^{-1}\right)$ in Peak 1. (iii) A small proportion of the flows may represent sites of low mass star formation and one region shows evidence of multiple flows which may indicate multiple low mass star formation within OMC1.
\end{abstract}

Key words. ISM: jets and outflows - ISM: structure - stars: formation - stars: circumstellar matter - ISM: kinematics and dynamics - ISM: molecules

\section{Introduction}

The Orion Molecular Cloud 1 (OMC1) $\left(D=460 \mathrm{pc}^{1}\right)$ is the closest highly active massive star-forming region. It has accordingly been studied over a large range of wavelengths from the radio to the X-ray (e.g. Genzel \& Stutzki 1989; Wright et al. 1996; O'Dell 2001; Chrysostomou et al. 2000; Garmire et al. 2000; Ferland 2001; Feigelson et al. 2002; Doi et al. 2002; O'Dell \& Doi 2003; Tan 2004) and has become the archetypal site for studies of star formation. Many features are accordingly very well-described, such as the detailed morphology of the HII region associated with $\theta^{1}$ Ori $\mathrm{C}$, the dominant source of ionizing radiation in the Trapezium (O'Dell 2001). Perhaps the principal significance of $\mathrm{OMC1}$ and its vicinity is that it has been shown to harbour upward of 1000 young low mass stars in the presence of a number of high mass stars (Hillenbrand 1997). For a general review of the Orion Nebula Cluster see O'Dell (2001).

The BN/IRc2 complex has an IR luminosity of $\sim 10^{5} L_{\odot}$ indicating the presence of embedded massive protostars in the

* Based on observations obtained at the Canada-France-Hawaii Telescope (CFHT) which is operated by the National Research Council of Canada, the Institut National des Sciences de l'Univers of the Centre National de la Recherche Scientifique of France, and the University of Hawaii.

$\star \star$ Tables A.1-A.3 are only available in electronic form at http: //www . aanda. org

$\star \star \star$ Visiting astronomer at the Canada-France-Hawaii Telescope, Mauna Kea, Hawaii.

1 We follow the example of Bally et al. (2000) and adopt a distance of $460 \mathrm{pc}$ based on the maser observations by Genzel et al. (1981). central parts of OMC1. Further evidence of embedded massive protostars is provided by the powerful outflows originating from the BN/IRc2 complex. The first, the "CO outflow" is a fast (30-100 $\mathrm{km} \mathrm{s}^{-1}$ ) bipolar outflow oriented NW-SE and was initially detected in CO by Kwan \& Scoville (1976) and Zuckerman et al. (1976). The energy of this flow is around $10^{47} \mathrm{erg}$ and it contains about $10 M_{\odot}$ of gas (Kwan \& Scoville 1976). The second outflow was first described by Genzel et al. (1981) based on proper motion studies of $\mathrm{H}_{2} \mathrm{O}$ masers. This outflow is oriented NE-SW, that is, perpendicular to the first outflow and is much slower at $\sim 18 \mathrm{~km} \mathrm{~s}^{-1}$. Properties of these outflows are reviewed in Genzel \& Stutzki (1989). Further outflow motion from the BN/IRc2 complex is present in the form of the Orion "bullets" (Axon \& Taylor 1984) and the associated "fingers" (Allen \& Burton 1993). The fingers lie principally to the NW of OMC1 but some are also visible to the SE (Kaifu et al. 2000). They are the wake of the fast-moving, diffuse clumps of gas, known as bullets, moving radially out from $\mathrm{OMC1}$ at speeds of several hundreds $\mathrm{km} \mathrm{s}^{-1}$ (Axon \& Taylor 1984; Burton 1997; Lee \& Burton 2000; Doi et al. 2002).

Two classes of models have been proposed to explain the overall structure of OMC1. These models involve either a wind, with shock instabilities (Stone et al. 1995; McCaughrean \& Mac Low 1997) or an explosive event (e.g. Allen \& Burton 1993; Doi et al. 2002), both of which create bright knots of fast flowing shocked gas. The physical origin of winds and/or explosions is not established. The outflows mentioned above appear to share a common area of origin in the central part of OMC1 near radio sources I and n (Menten \& Reid 1995). Source I is a 
deeply buried, massive star observed only in the radio. Source $n$ is a somewhat less massive star detected both in radio and IR (Greenhill et al. 2004a,b; Beuther et al. 2004). Evidence has been presented to suggest both source I and source $n$ as the driving force behind the slow outflow (Greenhill et al. 2004a,b; Shuping et al. 2004). Source I is also suggested as the source of the fast $\mathrm{CO}$ outflow based on a different interpretation of the data (Shuping et al. 2004; Bally \& Zinnecker 2005) and the role of source I remains unresolved.

The age of the two outflows is different; the slow outflow of $18 \mathrm{~km} \mathrm{~s}^{-1}$ has been active for at least 3000 years (Genzel et al. 1981) while the fast outflows are only about a thousand years old (Lee \& Burton 2000; Doi et al. 2002), assuming the same age for the $\mathrm{CO}$ outflow and the finger system. Recent studies of the motions of BN, source I and source n (Tan 2004; Rodríguez et al. 2005; Gómez et al. 2005) have shown that these massive stars passed within about an arcsecond of each other about 500 years ago. This has led to the suggestion that a cataclysmic event took place due to dynamic instabilities in this close passage, resulting in the ejection of one or more of the massive stars from the core of OMC1 and the launching of the finger-system (Bally \& Zinnecker 2005). The apparent time discrepancy here, 500 years for the close passage versus 1000 years for the age of the finger system, may be explained by uncertainties in the ages or by a deceleration of the finger system as it passed through Peak 1 (Bally \& Zinnecker 2005).

OMC1 contains a great deal of dense gas evidenced by the presence of $\mathrm{H}_{2} \mathrm{O}, \mathrm{OH}$, methanol and $\mathrm{SiO}$ masers, the latter near source I (Menten \& Reid 1995; Greenhill et al. 2004b) where densities of the order of $10^{9} \mathrm{~cm}^{-3}$ must locally prevail in order to sustain $\mathrm{H}_{2} \mathrm{O}$ masers (Yates et al. 1997). Shock models have shown that pre-shock densities of $10^{6} \mathrm{~cm}^{-3}$ are necessary to explain the observed $\mathrm{H}_{2}$ brightness (Vannier et al. 2001; Kristensen et al. 2003, 2006).

High densities coupled with the presence of outflows from massive stars make OMC1 a prime candidate for shock-induced star formation as the origin for the cluster of low mass stars in OMC1 (Hillenbrand 1997). However very little is known of the low mass protostellar population of the inner $1^{\prime} \times 1^{\prime} \mathrm{OMC} 1$. The best evidence of the presence of such a population is provided by the $L$-band data of Lada et al. (2004) and by the COUP X-ray data (Grosso et al. 2005) both of which show a significant number of embedded point sources.

We are concerned here exclusively with observation of vibrationally excited $\mathrm{H}_{2}$ emission in the $K$-band $(2-2.5 \mu \mathrm{m})$. High spatial resolution $K$-band images of IR emission from $\mathrm{H}_{2}$ in OMC1 using the HST (Stolovy et al. 1998; Chen et al. 1998; Schultz et al. 1999; Doi et al. 2002), the Canada-FranceHawaii Telescope (CFHT) (Gustafsson et al. 2003, hereafter G2003), the ESO $3.6 \mathrm{~m}$ (Vannier et al. 2001; Kristensen et al. 2003), the VLT (Lacombe et al. 2004) and other telescopes (e.g. McCaughrean \& Mac Low 1997; Schild et al. 1997; Kaifu et al. 2000) have provided the most recent evidence for the presence of fast flows and shocks at small scales in OMC1. The present work, covering the area shown in Figs. 1 and 2, concentrates in particular upon data displaying the dynamics of OMC1. $\mathrm{H}_{2} v=1-0 \mathrm{~S}(1)$ emission shows the gas in OMC1 as highly structured with many bright knots and there is ample evidence of much violent motion within OMC1 over a range of scales from $70 \mathrm{AU}$ to $3 \times 10^{4} \mathrm{AU}$ (Gustafsson et al. 2006a). Earlier work concerning gas dynamics (Chrysostomou et al. 1997; Salas et al. 1999, G2003) showed the presence of numerous gas flows with flow velocities varying from a few $\mathrm{km} \mathrm{s}^{-1}$ in relatively dense gas to several

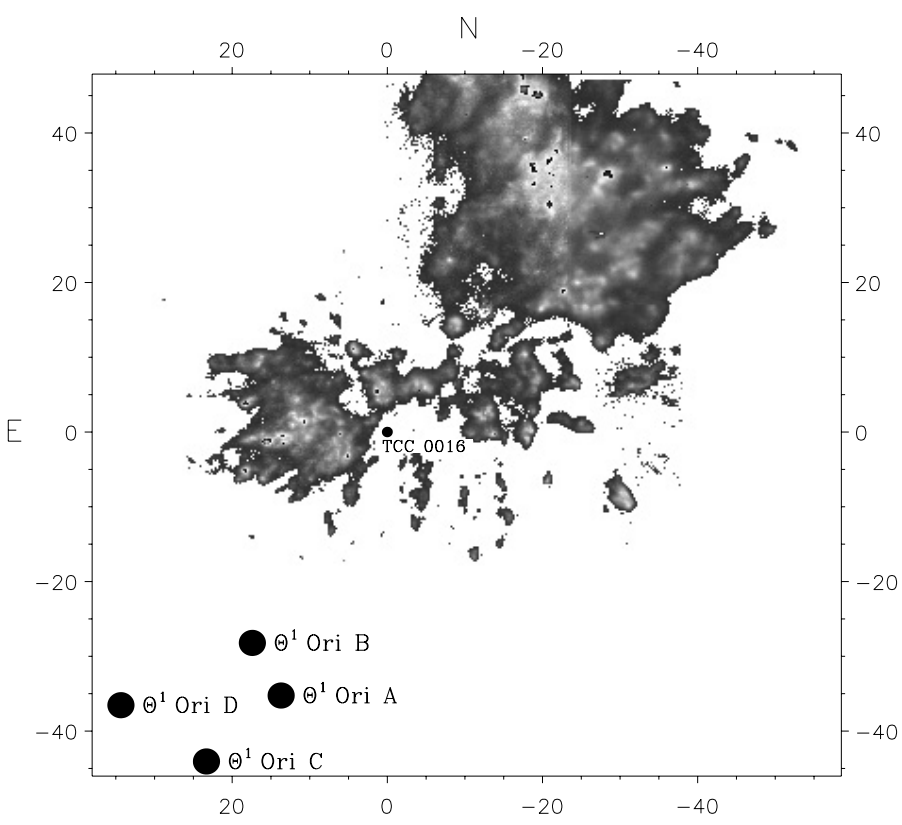

Fig. 1. The observed area relative to the Trapezium stars (large black dots). The grey scale image show emission brightness in the $\mathrm{H}_{2}$ $v=1-0 \mathrm{~S}(1)$ line. Axes are labelled in arcseconds. The star TCC0016, at coordinates $0^{\prime \prime} \mathrm{E}, 0^{\prime \prime} \mathrm{N}$ and marked with a small black dot, is used as a reference for coordinates throughout this work.

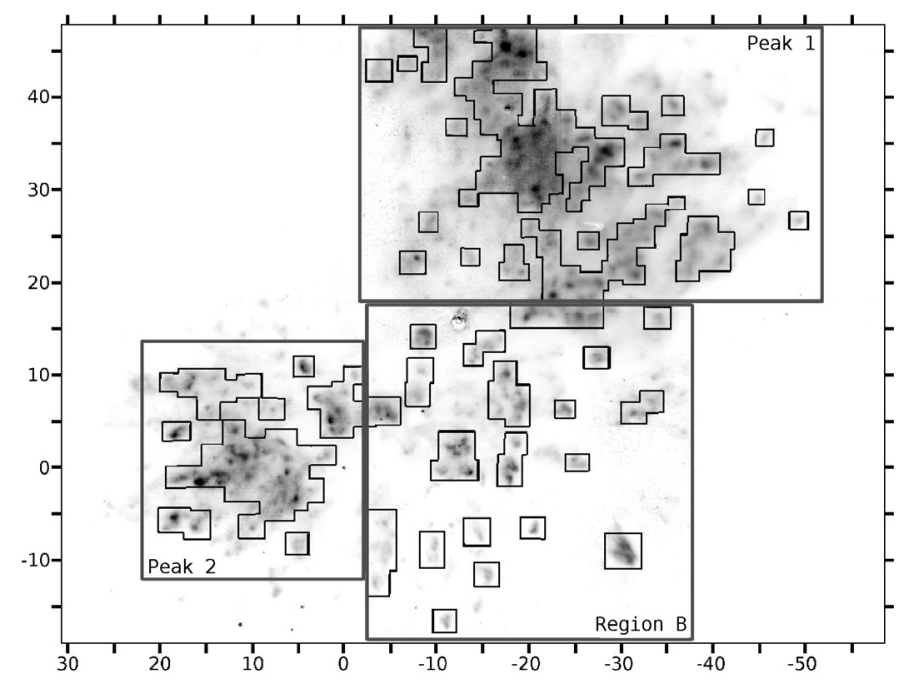

Fig. 2. The regions analysed in this work outlined as small overlapping black boxes. The gray-scale shows emission in the $\mathrm{H}_{2} v=1-0 \mathrm{~S}(1)$ emission line. Also shown as large black boxes is the naming convention for Peak 1, Peak 2 (Beckwith et al. 1978), and region B, the latter bounded by $-2^{\prime \prime}--40^{\prime \prime} \mathrm{E},-18^{\prime \prime}-17^{\prime \prime} \mathrm{N}$, relative to TCC0016. Axes are labelled in arcseconds.

hundred $\mathrm{km} \mathrm{s}^{-1}$ (Lee \& Burton 2000; Doi et al. 2002). The latter motions are associated with the finger-system.

The region studied in the present work (Fig. 1) covers the inner $1^{\prime} \times 1^{\prime}$ of $\mathrm{OMC1}$, excluding most of the well-studied outer part of the finger system. Thus the material in the present study encompasses the region that tends to be subject to denser, slower outflows, associated with massive outflows from the core of OMC1 and also to a smaller degree with possible local, low mass star formation.

We work here within the framework of the second class of the models described above, where an explosive event has 
ejected "shrapnel" to form the bullets shooting through Peak 1 (see Fig. 2 for nomenclature). The bright $\mathrm{H}_{2}$ emission in Peak 1 is then due mainly to the large scale energy input from the explosion which is expressed in part by shocks formed by bullets ploughing through the gas. There is also an important contribution due to a turbulent cascade of energy in OMC1, as described in detail in Gustafsson et al. (2006b), in which supersonic turbulence may give rise to shocked $\mathrm{H}_{2}$ emission. We also note that Gustafsson et al. (2006b) shows the presence of structure at a preferred scale of around $1000 \mathrm{AU}$ consistent with a protostellar population in OMC1.

G2003 provided an initial report of $\mathrm{H}_{2}$ emission data for OMC1 obtained with the GriF instrument on the CFHT. These data, containing information on both morphology and velocity, showed striking evidence for the presence of shocks within OMC1. This was provided through the clear association of gas motions of tens of $\mathrm{km} \mathrm{s}^{-1}$ with bright IR emission of $\mathrm{H}_{2}$. The novelty of these data is contained in the association of a very high spatial resolution of effectively $0.18^{\prime \prime}$ (80 AU) with an effective velocity discrimination as good as $1 \mathrm{~km} \mathrm{~s}^{-1}(3 \sigma)$ in regions of high emission brightness (see Sect. 2).

In the present work the GriF data are reported in detail. Whereas in G2003 19 regions were identified for study, 193 regions are included here. This affords a much broader perspective of the nature of flows within OMC1 and represents a detailed attempt at extracting the wealth of information contained within the GriF observations. New features are revealed through this extended study of the GriF results.

In Sect. 2 the observations and data reduction, described in detail in G2003, are briefly reviewed. Section 3 describes the criteria and methods used in selecting and analysing the 193 regions. The mechanism of $\mathrm{H}_{2}$ excitation is also briefly discussed. In Sect. 4 the data are presented in order to give a global view of the spatial distribution of radial flow velocities and the characteristics of various zones. Section 5 describes the use of data to establish the orientations of shocks within the plane of the sky. Then follows a consideration of possible current low mass star formation in OMC1. In Sect. 6 the origin of the outflow in region B and its spatial association with continuum sources in the IR and radio is discussed in detail.

\section{Observations and data reduction}

Observations made in the $\mathrm{H}_{2} v=1-0 \mathrm{~S}(1)$ emission line, which has a rest wavelength of $2.1212544 \mu \mathrm{m}$ (Bragg et al. 1982), are reported here. The data are used to determine a full radial velocity map of the gas in the central zone of OMC1. The total observed field is a $\sim 1^{\prime} \times 1^{\prime}$ area amalgamated from four fields, each $36^{\prime \prime} \times 36^{\prime \prime}$. The total field is centered approximately on the BN-object NW of the Trapezium stars (see Figs. 1 and 2). The data used in this work are the same as those used in G2003, and a detailed description of the data reduction process is given there. Here we give only a brief resumé.

The observations were performed on the night of December 5th 2000 at the $3.6 \mathrm{~m}$ CFHT, using the then new instrument GriF (Clénet et al. 2002). The GriF instrument combines the high spatial resolution of the PUEO adaptive optics (Rigaut et al. 1998) with spectral resolution provided by a Queensgate ET50WF Fabry-Perot interferometer (FP). The finesse of the Fabry-Perot is 104 and the resolution is $\lambda / \Delta \lambda \sim$ 2000 , that is, $150 \mathrm{~km} \mathrm{~s}^{-1}$. For each of the four fields the FP was used to scan through the $\mathrm{H}_{2}$ line in steps of $\sim 4.5 \times 10^{-4} \mu \mathrm{m}$ from a point in the far blue wing to a point in the far red wing, using 8 to 12 settings. The step size was chosen to provide adequate sampling of the instrumental profile. This procedure yields a data cube showing brightness in each position (pixel) for a series of wavelengths. A $\mathrm{H}_{2} v=1-0 \mathrm{~S}(1)$ interference filter of central wavelength $2.122 \mu \mathrm{m}$ and bandwidth of $0.02 \mu \mathrm{m}$ was inserted between the FP and the detector to prevent the superposition of different FP orders. For each field a single $400 \mathrm{~s}$ exposure was made. Reference stars for the AO system were TCC0016, Parenago 1819 and Parenago 1838. Data reduction included dark and bias subtraction, flat-fielding, bad pixel rejection, 2D wavelength correction and subtraction of the sky background. The latter was obtained from images made from data in the far wings of the line.

The spatial resolution of the data was $0.15^{\prime \prime}$, estimated from the FWHM of the point spread functions of stars in the field. The pixel scale was $0.035^{\prime \prime}$. The data were smoothed with a moving boxcar average over $3 \times 3$ pixels to increase the signal-to-noise with only a minor loss of spatial resolution. yielding a resolution of $0.18^{\prime \prime}$. To determine radial velocities in a specific position a cut was made through a data cube giving the count rate for each observed wavelength, that is, a line profile. This profile was then fitted with a Lorentzian since this represents the line profile of the FP (Clénet et al. 2002). In addition a line profile is also extracted from a data cube recorded with a spectral calibration lamp. This provides an absolute wavelength reference. Shifts between peaks for the fitted profiles for observational data and calibration data yield radial velocities, where velocities are obtained from peaks in fitted profiles. As described in G2003, fits could be performed with very high precision for high $\mathrm{S} / \mathrm{N}$ data and the instrument showed a very accurately symmetrical and Lorentzian characteristic form.

Due to the high signal-to-noise ratio in the data we obtain a velocity resolution as good as $\pm 1 \mathrm{~km} \mathrm{~s}^{-1}(3 \sigma)$ in one region in Peak 2, flow 2-10, see Table A.2. 25\% of regions are sufficiently bright that the velocity uncertainty is better than $1.5 \mathrm{~km} \mathrm{~s}^{-1}(3 \sigma)$ and for $80 \%$ of regions the figure is $\pm 2 \mathrm{~km} \mathrm{~s}^{-1}(3 \sigma)$. Velocities of the gas surrounding regions of flow typically have uncertainties of 3-4 $\mathrm{km} \mathrm{s}^{-1}$. A detailed account of the errors associated with velocity determination may be found in Gustafsson et al. (2006b). An empirically determined expression for the standard deviation in the velocity in $\mathrm{km} \mathrm{s}^{-1}$, a little modified from Gustafsson et al. (2006b), is given by

$\sigma=-9.37+3.05 \exp (-I \cdot 6.93)+10.11 \exp (-I \cdot 0.026)$

where $I$ is the brightness in counts pixel ${ }^{-1} \mathrm{~s}^{-1}$ for the peak emission associated with the Lorentzian fit. For example a typical count rate of 300 in a 400 s exposure, corresponding to $I=0.75$, yields a $3 \sigma$ value of $\sim 1.7 \mathrm{~km} \mathrm{~s}^{-1}$.

Resulting velocity data show velocity profiles of a clear regular structure, shown for example in Fig. 3, characterised by welldefined localised peaks in the velocity. A specific example in the SE region was given in G2003, Fig. 2, and illustrates the high accuracy with which relative velocities in close lying regions may be obtained.

Because of the $150 \mathrm{~km} \mathrm{~s}^{-1}$ resolution of the $\mathrm{FP}$, the Lorentzian fit at a given point cannot show details in the line profile significantly smaller than this resolution. The velocity determined from the fits is therefore a brightness weighted average velocity of the gas in this direction rather than a resolved line profile. Such a profile could only be achieved by high intrinsic spectral resolution observations such as those of Chrysostomou et al. (1997). The uncertainties in velocities cited above are statistical uncertainties resulting only from noise and cannot take into account any effects of different objects lying in the same line-of-sight but with different median 


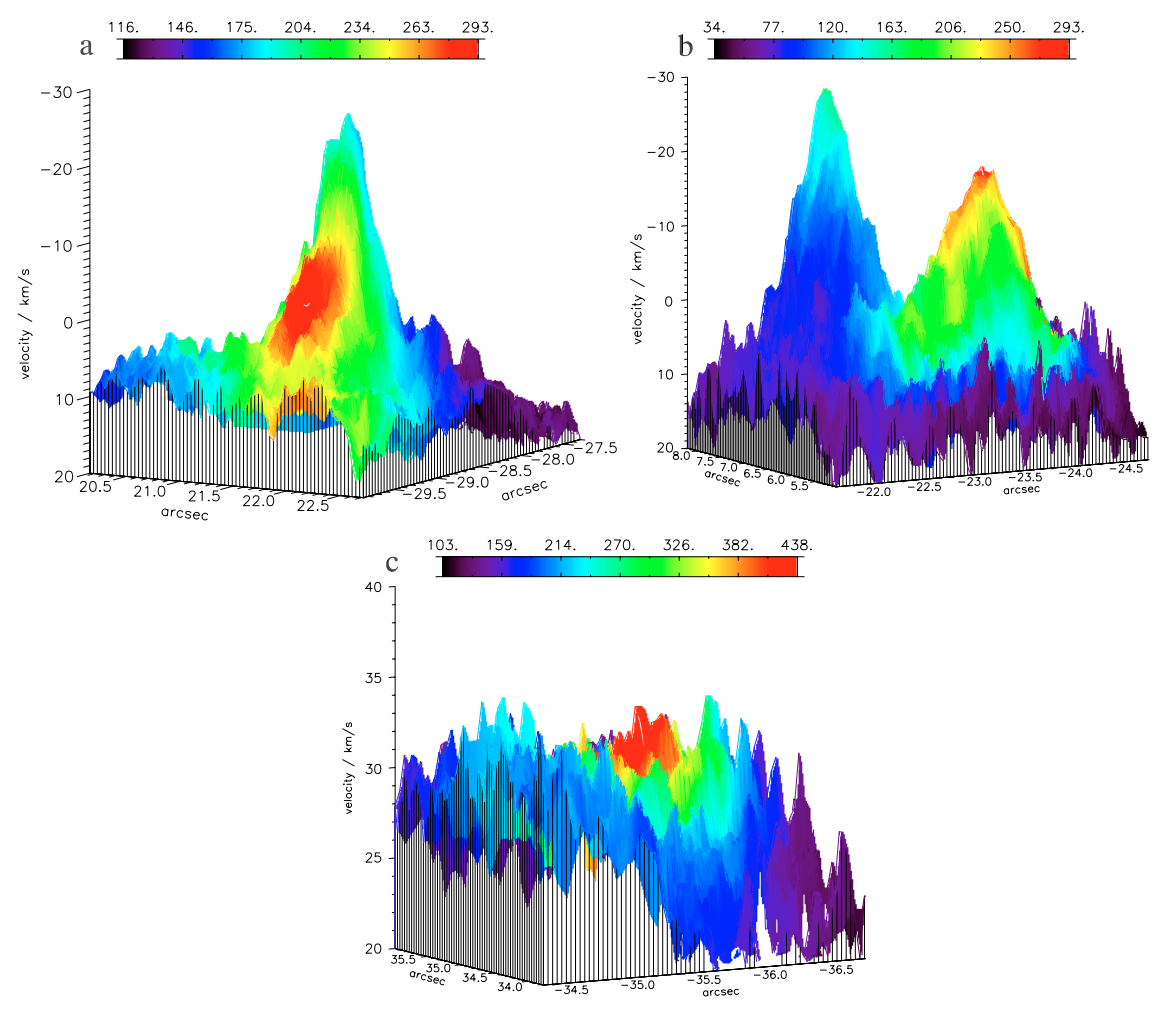

Fig. 3. a) Flow No. 1-61: see Table A.1. The vertical axis shows $v_{\mathrm{lsr}}$ in $\mathrm{km} \mathrm{s}^{-1}$. Brightness, in counts per $400 \mathrm{~s}$, is shown on the bar above the picture. The plane of the sky is shown in arcsec, relative to TCC0016. b) Flows No. B44, B-45 and B-46: see Table A.3. Otherwise as in a). c) Flow No. 1-74: see Table A.1. This is an example of a feature which shows no clear velocity peak exceeding $5 \mathrm{~km} \mathrm{~s}^{-1}$.

velocities. In this connection less than $2 \%$ of the profiles are double peaked. Where this was found the velocity of the brighter of the two peaks was chosen. As discussed in detail in G2003, our procedures thus reject weaker objects and are ill-suited to the detection of relatively faint bullets in the field. We are aware of two cases of such faint objects superposed on brigher, slower moving features: HH208 (Axon \& Taylor 1984; O'Dell et al. 1997), and that mentioned in G2003. In fact we detect a low velocity component of HH208, object B-42, Table A.3. Very high velocity objects $\left(>150 \mathrm{~km} \mathrm{~s}^{-1}\right)$ are also rejected through the limited range of the velocity channels.

The above figures for velocity and spatial resolution may be compared with the high inherent spectral resolution of $14 \mathrm{~km} \mathrm{~s}^{-1}$ in Chrysostomou et al. (1997) and $24 \mathrm{~km} \mathrm{~s}^{-1}$ in Salas et al. (1999), where data were recorded with a spatial resolution between $1.5^{\prime \prime}$ and $2.0^{\prime \prime}$.

We have calculated the mean velocity of all our data and then shifted all our values such that this mean becomes $12 \pm$ $6 \mathrm{~km} \mathrm{~s}^{-1}$. Thus figures of velocity quoted, save those for flow velocities, are effectively in the local standard of rest $\left(v_{\text {lsr }}\right)$, since the value of $12 \pm 6 \mathrm{~km} \mathrm{~s}^{-1}$ is consistent with the $v_{\mathrm{lsr}}$ given by data in Chrysostomou et al. (1997), Salas et al. (1999) and O'Dell (2001). This adjustment of the velocities is however not material to this work, as we are concerned with relative velocities over small distances of typically a few arcseconds. For further details on observations and data reduction see G2003 and references therein.

Throughout this paper (save in Fig. 12) all positions are given in arcseconds east and north relative to the star TCC0016, which itself is situated at $05^{\mathrm{h}} 35^{\mathrm{m}} 14^{\mathrm{s}} .91,-05^{\circ} 22^{\prime} 39^{\prime} \cdot 31$ (J2000).

\section{Data analysis}

\subsection{Identification and characterization of emitting zones}

Data for the 193 regions analysed here, are collated in Tables A.1-A.3 (available in the online version). All flows are designated by number in these tables. The tables each refer to one of the three regions into which the full region has been subdivided, namely Peak 1, Peak 2 and region B (see Fig. 2 for nomenclature).

Features were identified on the basis of their brightness and our concomitant ability to locate boundaries for any feature. Thus all features show a well-defined localized increase in brightness compared to the surrounding gas. Each of these features has been examined for evidence of a local gas flow. This was done by making 3D-images of each region, where these images show radial velocity and emission brightness as a function of the position in the plane of the sky. Two illustrative examples of features exhibiting flows are shown in Figs. $3 a$ and $b$, where the axes labelled in arcseconds give the position in the plane of the sky and the $z$-axis shows the radial velocity of the gas. A colour code associated with every image shows the observed brightness as the number of counts per exposure of $400 \mathrm{~s}$. This can be converted to brightness through the use of the value of $3.0 \pm 0.15 \times 10^{-5} \mathrm{~W} \mathrm{~m}^{-2} \mathrm{sr}^{-1}$ found in Vannier et al. (2001) for the brightest region observed here ${ }^{2}$. Using this value it is found that a count of 100 over the $400 \mathrm{~s}$ exposure corresponds to $\sim 3.5 \times 10^{-6} \mathrm{~W} \mathrm{~m}^{-2} \mathrm{sr}^{-1}$. This conversion may be applied to all regions (and all figures in this work) with the proviso that systematic errors may arise through differing conditions of airmass and of the atmosphere prevailing at the times of observation.

Figure 3 a clearly shows a very localized increase in the radial velocity ${ }^{3}$ and the brightness. The typical extent of the areas that show this phenomenon of strong localised brightness is $0.5^{\prime \prime}-1^{\prime \prime}$ in all the 193 regions identified in the data. All regions are therefore well resolved, given a spatial resolution of $0.18^{\prime \prime}$.

\footnotetext{
2 The brightest region is in Peak 2 at coordinates $15.82^{\prime \prime} \mathrm{E},-1.61^{\prime \prime} \mathrm{N}$, see Table A.2, flow No. 2-10.

${ }^{3}$ In the remainder of this article, velocity should be taken to mean the radial velocity unless otherwise specified.
} 
Data for each region have been characterised in terms of the following properties:

(i) the maximum velocity relative to the surrounding gas;

(ii) the maximum brightness;

(iii) the displacement in the plane of the sky between the maxima in velocity and brightness.

(i) The maximum velocity of the flow relative to the surrounding gas is found by taking the maximum velocity recorded in the region of interest and subtracting the average velocity of the surrounding gas. The latter may be estimated with a typical uncertainty of $3-4 \mathrm{~km} \mathrm{~s}^{-1}$ (see Sect. 2). The resulting flow velocity contains this uncertainty. Thus these velocities are less accurately determined than relative velocities between adjacent bright regions. Using Fig. 3a as an example, the velocity of the surrounding gas is $8 \pm 3 \mathrm{~km} \mathrm{~s}^{-1}$. The tip of the velocity peak is at $-24 \pm 1 \mathrm{~km} \mathrm{~s}^{-1}$ and thus the flow velocity relatively to the surroundings is $-32 \pm 4 \mathrm{~km} \mathrm{~s}^{-1}(3 \sigma)$.

In 64 out of the 193 regions there is an increase in brightness similar to that found, for example, in Figs. $3 a$ and b, but with no corresponding velocity structure. An example is shown in Fig. 3c. These features will be referred to as features with weak radial velocity components (WRVs). Figure 3c, which is a typical such object, demonstrates that velocity variations are of the order of less than $5 \mathrm{~km} \mathrm{~s}^{-1}$, with no clear velocity structure. This is therefore used as a definition of WRVs, which are defined as those objects with a localized maximum in brightness but which show no velocity excursions from the surrounding gas greater than $5 \mathrm{~km} \mathrm{~s}^{-1}$. WRVs according to their brightness and extent may arise from flows close to the plane of the sky. However, some of these features could also be bright photodissociation regions (see Sect. 3.2).

(ii) The maximum brightness is the highest recorded brightness within the confines of any region after the $3 \times 3$ boxcar smoothing mentioned in Sect. 2.

(iii) Spatial displacements of brightness and velocity features are listed in Tables A.1-A.3. There exists a strong spatial correlation between the local peaks in brightness and velocity: the distance in the plane of the sky between the two peaks is typically less than the extent of either peak. That is, they overlie each other in the great majority of cases, with the maximum in velocity lying within the area where the emission associated with the flow is more than twice the background emission. Figures $3 \mathrm{a}$ and $b$ illustrate this general property that features of velocity and brightness are closely associated in the plane of the sky.

\subsection{The origin of the $\mathrm{H}_{2}$ emission}

The mechanism by which the $\mathrm{H}_{2}$ emission is formed is briefly discussed here. This has already been considered in Vannier et al. (2001), Kristensen et al. (2003) and G2003. Vannier et al. (2001) described in detail, using the shock models of Wilgenbus et al. (2000), how the brightest regions in the Peak 2 could arise from magnetic (C-type) shock compression of dense gas at pre-shock densities around $10^{6} \mathrm{~cm}^{-3}$ by shocks travelling at $30 \mathrm{~km} \mathrm{~s}^{-1}$. Le Bourlot et al. (2002); Flower et al. (2003); Kristensen et al. (2003, 2006) expanded on these results and showed that C-type shocks of typically $25 \mathrm{~km} \mathrm{~s}^{-1}$ impinging on dense gas $\left(>10^{6} \mathrm{~cm}^{-3}\right)$ readily produce the brightest emission $\left(>10^{-5} \mathrm{~W} \mathrm{~m}^{-2} \mathrm{sr}^{-1},>300\right.$ counts per $\left.400 \mathrm{~s}\right)$ while photodissociation regions (PDRs) and J-type shocks make smaller contributions (see below). Using shock models and limiting their investigations to Peak 2, Vannier et al. (2001) sought to find if quantitative evidence could be established that shocks have collected enough gas and compressed it sufficiently to overcome the Jeans criteria. This work was therefore directed towards discovering if shock-induced star formation is an active mechanism. They found that in the brightest region in Peak $2\left(15.8^{\prime \prime} \mathrm{E}\right.$ $\left.-1.6^{\prime \prime} \mathrm{N}\right)$ the density was high enough to overcome the Jeans criteria over a region containing about $0.15 M_{\odot}$.

Shock-induced star formation is envisaged to take place on a timescale of $10^{4-5}$ years assuming free-fall collapse. Therefore any presently existing protostars cannot be associated with the current outflows, since these are too young. Shocks which may have induced protostar formation must have passed through the medium at a period $10^{4-5}$ years ago and are now no longer in evidence.

Both Kristensen et al. (2003) and G2003 addressed the question of the relative importance of shocks and PDRs in yielding the observed $\mathrm{H}_{2}$ emission brightness. $\theta^{1}$ Ori $\mathrm{C}$ generates a UV field, at around $100 \mathrm{~nm}$, more than $10^{5}$ times greater than the standard interstellar field. Detailed models for $\mathrm{H}_{2}$ emission have been devised, for example in Störzer \& Hollenbach (1999), for UV fields of this intensity falling upon dense gas, including advection and photoevaporation (Henney \& O'Dell 1999). These models show that the maximum contribution to emission brightness in the $\mathrm{H}_{2} v=1-0 \mathrm{~S}(1)$ line from PDRs does not exceed a few times $10^{-6} \mathrm{~W} \mathrm{~m}^{-2} \mathrm{sr}^{-1}$, given a line-of-sight normal to the PDR. For this geometry, the PDR contribution to the brightness is therefore no more than $10 \%$ to $15 \%$ of the maximum observed here. Note that with a few exceptions all of the areas examined in this work have a maximum brightness which is $25 \%$ or more of the observed maximum brightness. Thus all regions show clear evidence of flows with a brightness above the $15 \%$ limit for PDR contributions. The issue of a PDR contribution is re-examined briefly in the discussion of weak radial velocity components (WRVs) in Sect. 5.3. The conclusion presently is that the brightness due to photoexcitation is a generally minor though non-negligible contributor to the $\mathrm{H}_{2}$ emission observed here (Kristensen et al. 2003).

\section{Results}

To illustrate the nature of the data collated in Tables A.1-A.3, all features have been plotted in Fig. 4, where each circle marks an individual feature. The radius of the circle is proportional to the maximum brightness, and the colour shows whether features are red-shifted (red circles), blue-shifted (blue circles) or if WRVs were found to be present (yellow circles).

\subsection{Flow velocities}

Figure 5 shows the number of flows for a given velocity for Peaks 1 and 2 and region B. Several properties of the flows are apparent:

(i) The fraction of red-shifted flows compared to blue-shifted is similar in Peaks 1 and 2, with 33\% in Peak 1 and $45 \%$ in Peak 2. There are thus overall fewer red-shifted than blueshifted flows. The detection of bright $\mathrm{H}_{2}$ emission is influenced by the local dust optical depth. The near-IR may be strongly obscured in OMC1 (Rosenthal et al. 2000). Since the densities may exceed $10^{7} \mathrm{~cm}^{-3}$, one hundred to a few hundred $\mathrm{AU}$ depth of material is sufficient to obscure $\mathrm{H}_{2}$ emission. Thus material is preferentially observed emerging from the obscuring dust, rather than retreating into it, that is, blue shifted flows are expected to be detected more readily than red shifted, as found. 


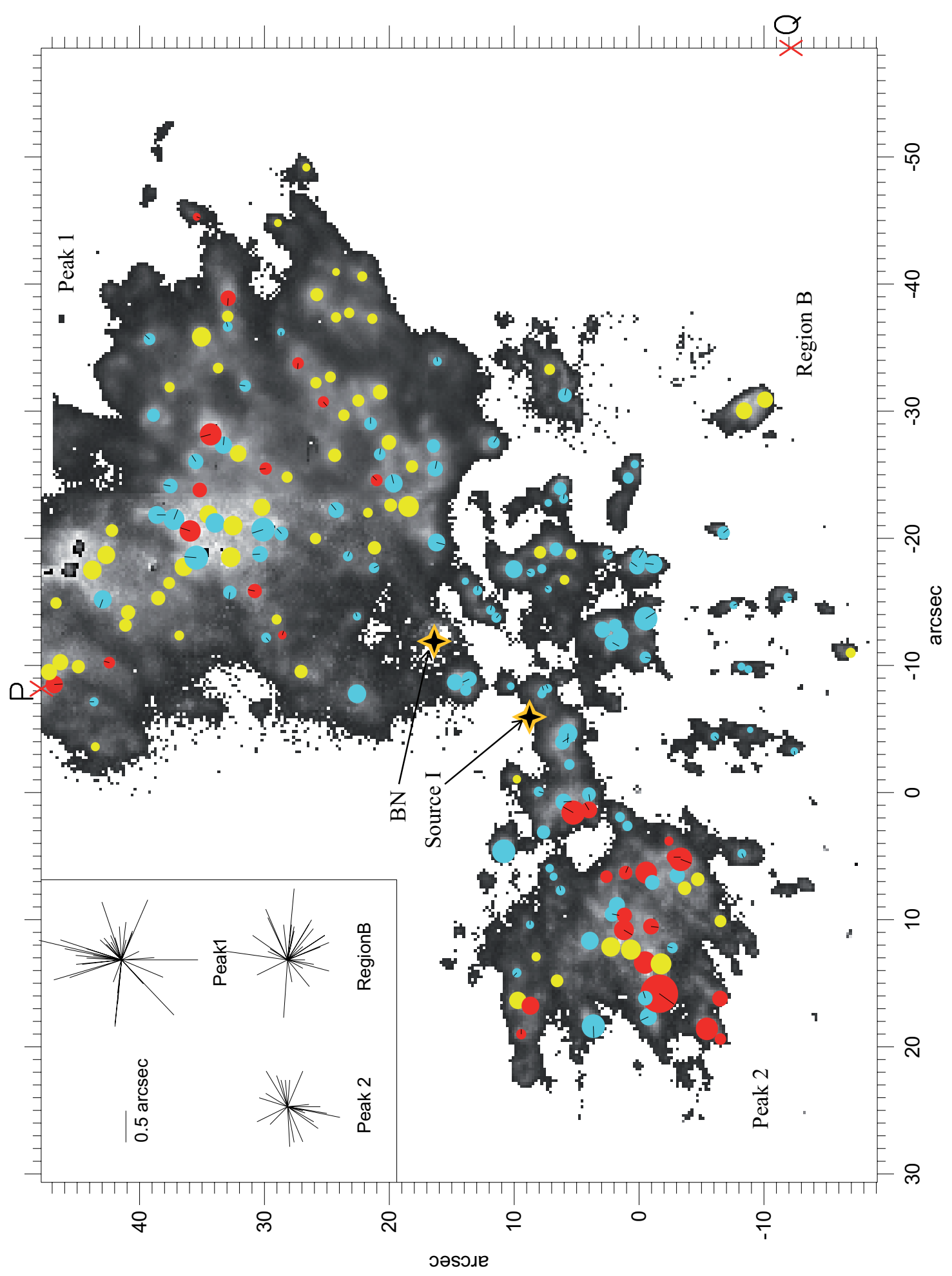

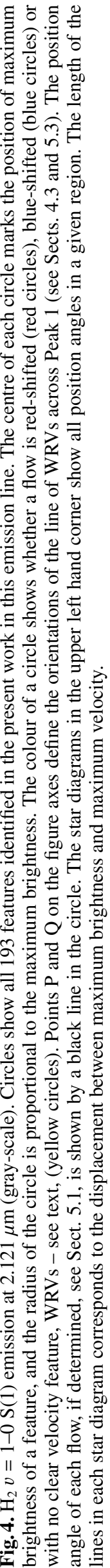



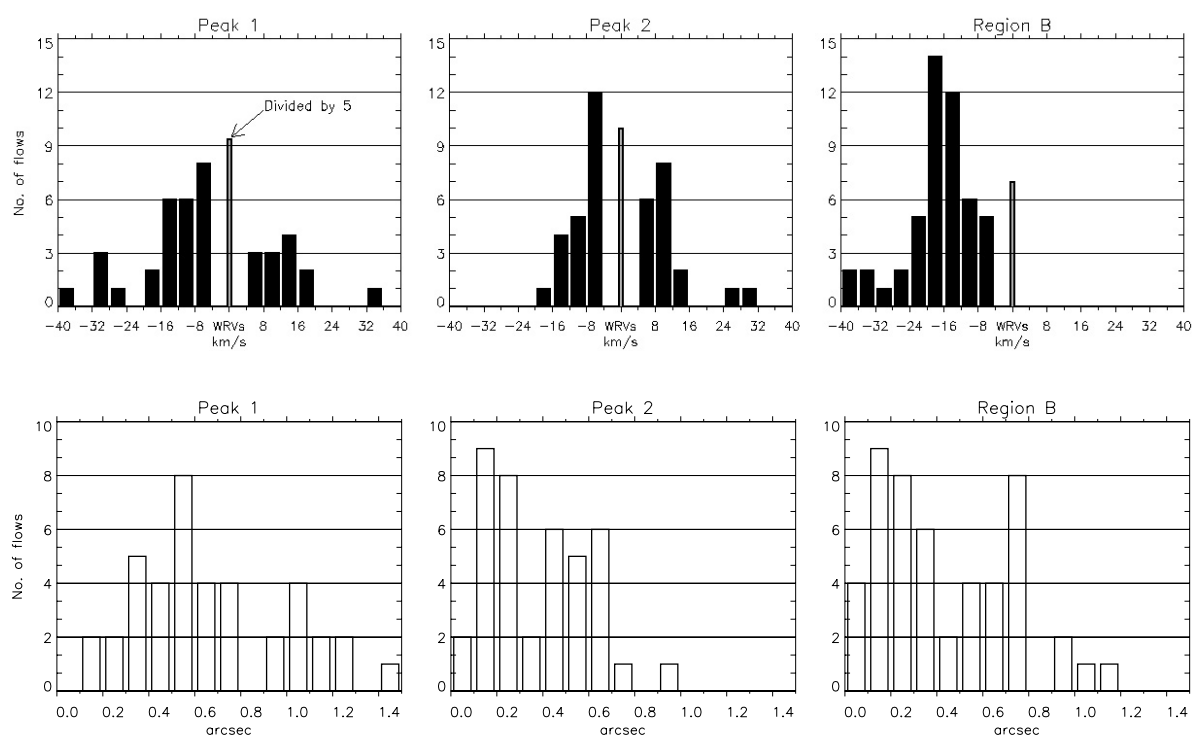

Fig. 5. Histograms showing the number of flows with a given radial velocity relative to the velocity of the immediate surroundings in each of the three main regions. Velocities are binned over $4 \mathrm{~km} \mathrm{~s}^{-1}$. Weak radial velocity features (WRVs) are by definition binned over $10 \mathrm{~km} \mathrm{~s}^{-1}$ (see Sect. 3.1). WRVs are shown as gray-shaded bars.

Fig. 6. Numbers of flows with a given displacement between the maxima in brightness and velocity for each of the three main regions. (ii) Compared to Peak 2, Peak 1 has a large over-representation of WRVs, as evidenced by Fig. 5a and as is clear from Fig. 4. WRVs in Peak 1 may have a different origin from other features in Peaks 1 and 2, possibly arising from an outflow associated with the BN-IRc2 region and/or with a possible PDR contribution. This is discussed in Sects. 4.3 and 5.3 .

(iii) The total number of flows in Peak 1 and Peak 2 is 40 in each case, excluding WRVs. Since Peak 2 is less than half the size of Peak 1, the density of flows is correspondingly larger in Peak 2.

(iv) In region B all flows are blue-shifted and the velocities are distributed around an average value of $\sim-18 \mathrm{~km} \mathrm{~s}^{-1}$ with a spread of $8 \mathrm{~km} \mathrm{~s}^{-1}$.

(v) For both Peak 1 and Peak 2 the red- and blue-shifted flows appear clumped around $+10 \mathrm{~km} \mathrm{~s}^{-1}$ and $-10 \mathrm{~km} \mathrm{~s}^{-1}$ respectively. Alternatively there may be a single distribution of flows around zero velocity. Note that the WRVs in Fig. 5 are binned over $10 \mathrm{~km} \mathrm{~s}^{-1}$, whereas velocities are binned over $4 \mathrm{~km} \mathrm{~s}^{-1}$.

(vi) Flow velocities lie between a few $\mathrm{km} \mathrm{s}^{-1}$ and an upper limit of $\sim 35-40 \mathrm{~km} \mathrm{~s}^{-1}$ for the radial velocity. With regard to the hypothesis that some of the flows may be associated with protostellar outflows, these figures agree with results from Davis et al. (2001) where flow velocities of 5-40 $\mathrm{km} \mathrm{s}^{-1}$ are recorded for a sample of 9 protostellar zones. The upper limit is consistent with results in Le Bourlot et al. (2002) for the critical velocity at which a $\mathrm{C}$-type shock collapses to J-type, in gas of pre-shock density $10^{5}-10^{6} \mathrm{~cm}^{-3}$.

Our general conclusion at this stage is that $\mathrm{H}_{2}$ emission in Peaks 1 and 2 represents a combination of outflows from massive stars within OMC1, which dominate on account of their massive energy injection, combined with local flows, perhaps due to protostars or local supersonic turbulence.

\subsection{Displacement of velocity and $\mathrm{H}_{2}$ emission brightness}

Figure 6 shows the distribution of displacements between associated maxima in brightness and in velocity for each region. We find that for $2 / 3$ of the flows the displacement between maximum velocity and maximum brightness is $\leq 0.6^{\prime \prime}$, and less than $10 \%$ show a displacement of $\geq 1.0^{\prime \prime}$. This should be compared to the typical extent of a peak in velocity or brightness which is of the order of $0.5^{\prime \prime}-1^{\prime \prime}$. Data in Fig. 6 quantify the earlier statement that a strong correlation exists between the positions of maxima in brightness and velocity (Sect. 3.1), implying that these are phenomena which are directly connected.

\subsection{Spatial distribution of flows with weak radial velocity components (WRVs)}

Several features are apparent in the spatial distribution of WRVs.

(i) WRVs comprise only a small proportion of the total number of flows in Peak 2 while in Peak 1 they outnumber all of the flows identified.

(ii) As explained in Sect. 3.1 the appearance of WRVs in shape, extent and maximum brightness is very similar to that of non-WRVs. If on this basis we assume that the WRVs are shocks moving in or near the plane of the sky, we find that this implies an inhomogeneous distribution of motion within Peak 1. This may be shown as follows.

If the general flow pattern in Peak 1 were homogeneous in three dimensions, then an estimate may be made of the number of flows that are expected to fit into the category of WRVs.

Given that typical shock speeds in OMC1 are $\sim 30 \mathrm{~km} \mathrm{~s}^{-1}$ (as suggested by results in Vannier et al. 2001; Kristensen et al. 2003, 2006), the limit imposed on the radial velocity to define a flow as a WRV, $v_{\text {radial }}<\left|5 \mathrm{~km} \mathrm{~s}^{-1}\right|$, corresponds to flows moving within approximately $\pm 10^{\circ}$ of the plane of the sky. This is illustrated in Fig. 7. For a homogeneous distribution of flow direction, the number of flows designated as WRVs relative to the total number of flows should equal the relative area of a band on a sphere around the equator from $-10^{\circ}$ to $+10^{\circ}(=\pi / 18 \mathrm{rad}$.):

$\frac{\begin{array}{l}\text { No. of flows in the } \\ \text { plane of the sky }\end{array}}{\text { total No. of flows }}=\frac{\int_{-\pi / 18}^{\pi / 18} 2 \pi \cos \theta \mathrm{d} \theta}{4 \pi} \approx 0.17$.

Correspondingly the non-WRVs should comprise $83 \%$ of the flows. As noted in Sect. 4.1 there are more blue- than red-shifted flows. If we assume that the small excess of blue over red-shifted flows is due to extinction, then a good estimate of the true total number of flows is given by twice the number of blue-shifted flows, rather than simply of sum of 


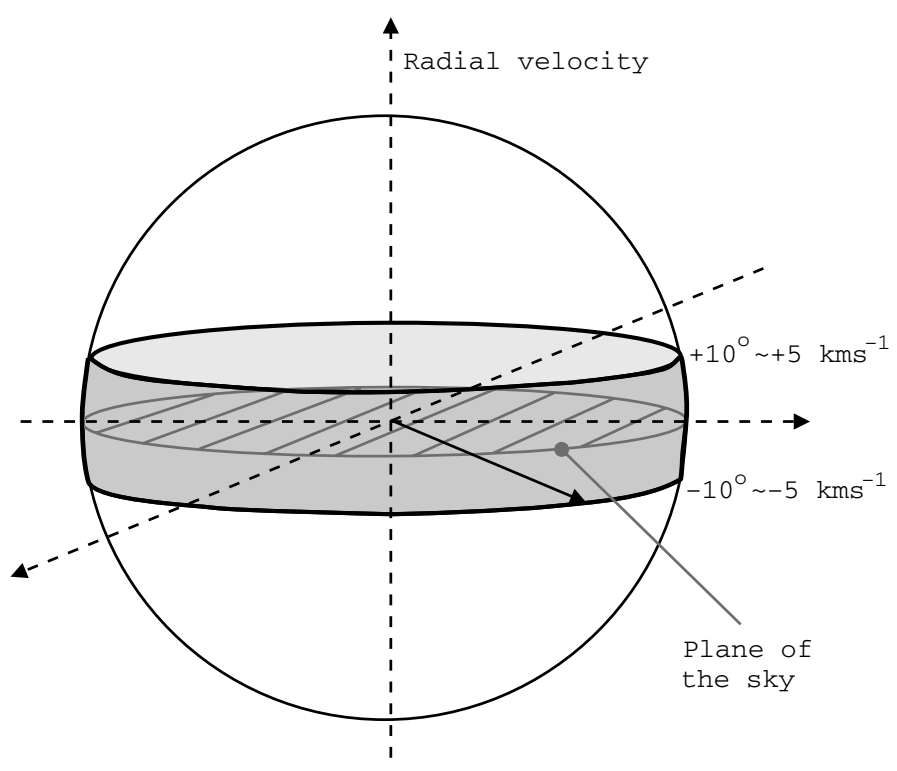

Fig. 7. Illustrating the ratio of the number of flows showing less than $5 \mathrm{~km} \mathrm{~s}^{-1}$ radial velocity (WRVs) to the total number of flows: see Sect. 4.3 and Eq. (2).

red and blue-shifted objects. Thus we estimate that the total number of non-WRVs to be 44 in Peak 2. The corresponding number of WRVs in a model of homogeneously directed flows should then be 9 . This agrees well with the 10 examples observed. The same model applied to Peak 1 suggests that $\sim 11$ WRVs should be seen. However 47 WRVs are observed in Peak 1. The above results do not change significantly if a typical shock speed of 25 or $20 \mathrm{~km} \mathrm{~s}^{-1}$ is assumed instead of $30 \mathrm{~km} \mathrm{~s}^{-1}$. Thus the flows do not form a spatially homogeneous sample in Peak 1 but lie preferentially in the plane of the sky and appear disproportionately as WRVs.

(iii) The data in Fig. 4 show not only that there is a considerable excess of WRVs in Peak 1 but also that there is the appearance of a concentration of WRVs along a direction on the eastern side of Peak 1 from $-8^{\prime \prime}$ E, $47^{\prime \prime} \mathrm{N}$ to $-31^{\prime \prime} \mathrm{E}, 21^{\prime \prime} \mathrm{N}$ along the line connecting points $\mathrm{P}$ and $\mathrm{Q}$ in Fig. 4. This line (or slit) is $\sim 30^{\prime \prime}$ long but only a few arcseconds wide. To assess whether this is a genuine feature of the data or merely a trick of the eye, a slit of length $30^{\prime \prime}$ and width $2.5^{\prime \prime}$ has been placed at all positions and for each position at all angles over the data for Peak 1 in Fig. 4. In each case the number of WRVs has been counted within the slit. Figure 8 shows the number of WRVs in the slit at the optimum angle in the sky to pick up the maximum number of WRVs, that is, at a position angle of $220^{\circ}$, as a function of the perpendicular distance of the slit from TCC0016. This corresponds to the slit being placed along lines parallel to the line connecting points $\mathrm{P}$ and $\mathrm{Q}$ in Fig. 4. The presence of a very clear maximum maximorum indicates that there is a genuine concentration of WRVs at the position designated.

(iv) A further property distinguishes the WRVs in the front identified in Fig. 8 from all other features. An investigation of the morphology of the $\mathrm{H}_{2}$ emission in the WRVs in the front reveals that $\sim 2 / 3$ of these objects show a very much more broken up morphology than that typically seen in features elsewhere in Peak 1 and Peak 2, for which emission is clearly associated with radial motion. Moreover, even in other WRVs, outside of this concentration, such a broken up morphology is only seen in $10-15 \%$ of the cases. The

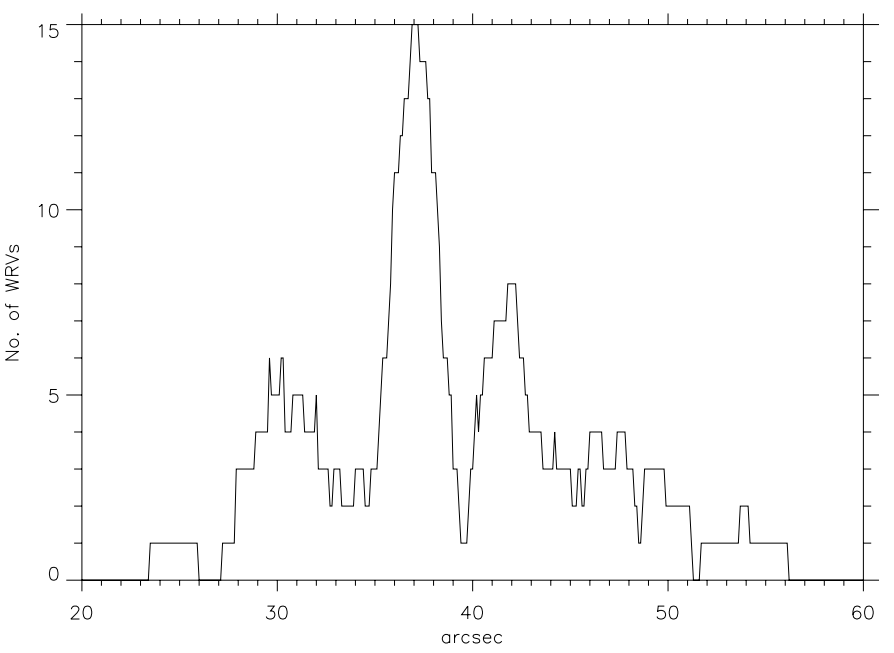

Fig. 8. The number of WRVs in a slit placed across Peak 1 at a position angle of $220^{\circ}$ as a function of perpendicular distance between the slit and TCC0016. The position of the peak at 37" corresponds to a slit placed along the line connecting points $\mathrm{P}$ and $\mathrm{Q}$ in Fig. 4.

broken-up morphology associated with the concentration of WRVs is illustrated in Fig. 9b. This may be compared to the relatively uniform increase towards a single maximum seen in most other WRV objects such as the WRV shown in Fig. 9a. A possible origin of broken-up structure is briefly considered in Sect. 5.3.

\subsection{Grouping of flows}

In the present section we exclude WRVs and consider how the remaining objects for which radial velocities can be measured are spatially distributed. Figure 10 shows how many blueand red-shifted flows lie within a distance of $1200 \mathrm{AU}\left(2.6^{\prime \prime}\right)$ from any given point, where each flow is represented by a circle of radius $1200 \mathrm{AU}$. A radius of $1200 \mathrm{AU}$ was chosen because this covers the typical distances between individual objects in binary or multiple star formation, e.g. Bate et al. (2003); Delgado-Donate et al. (2004). This is also the scale where Gustafsson et al. (2006b) find very clear deviations in their structure functions, indicative of a preferred scale. Figure 10 illustrates whether the flows have a tendency to be grouped together or are spaced equally across the entire area. Peak 1 and Peak 2 show different character. In Peak 1 the flows are distributed rather evenly across the entire area, with only one position showing a large number of flows in a small area, around the position $-22^{\prime \prime}$ E, $36^{\prime \prime}$ N. In Peak 2, flows are more grouped with some areas showing 6-8 flows within an area of $\sim 2500 \mathrm{AU}$ in diameter around positions $1^{\prime \prime} \mathrm{E}, 5^{\prime \prime} \mathrm{N}$ and $9^{\prime \prime} \mathrm{E}, 1^{\prime \prime} \mathrm{N}$. Taken together with the observation, reported in Sect. 4.1, that the spatial density of flows is approximately twice as high in Peak 2 as in Peak 1, these results imply that Peak 2 is a more active region than Peak 1.

\subsection{Further characteristics of the outflow in region $B$}

As noted, all flows are blue-shifted in region B and the mean of the velocities is significantly greater than in Peaks 1 and 2 . Moreover region B contains, among the 49 objects in total, five clear examples of bow shocks, based on the morphology of the $\mathrm{H}_{2}$ emission. An example is displayed in Fig. 11, the left-hand panel of which shows a NAOS-CONICA adaptive optics image obtained with the VLT (Lacombe et al. 2004), lacking velocity 


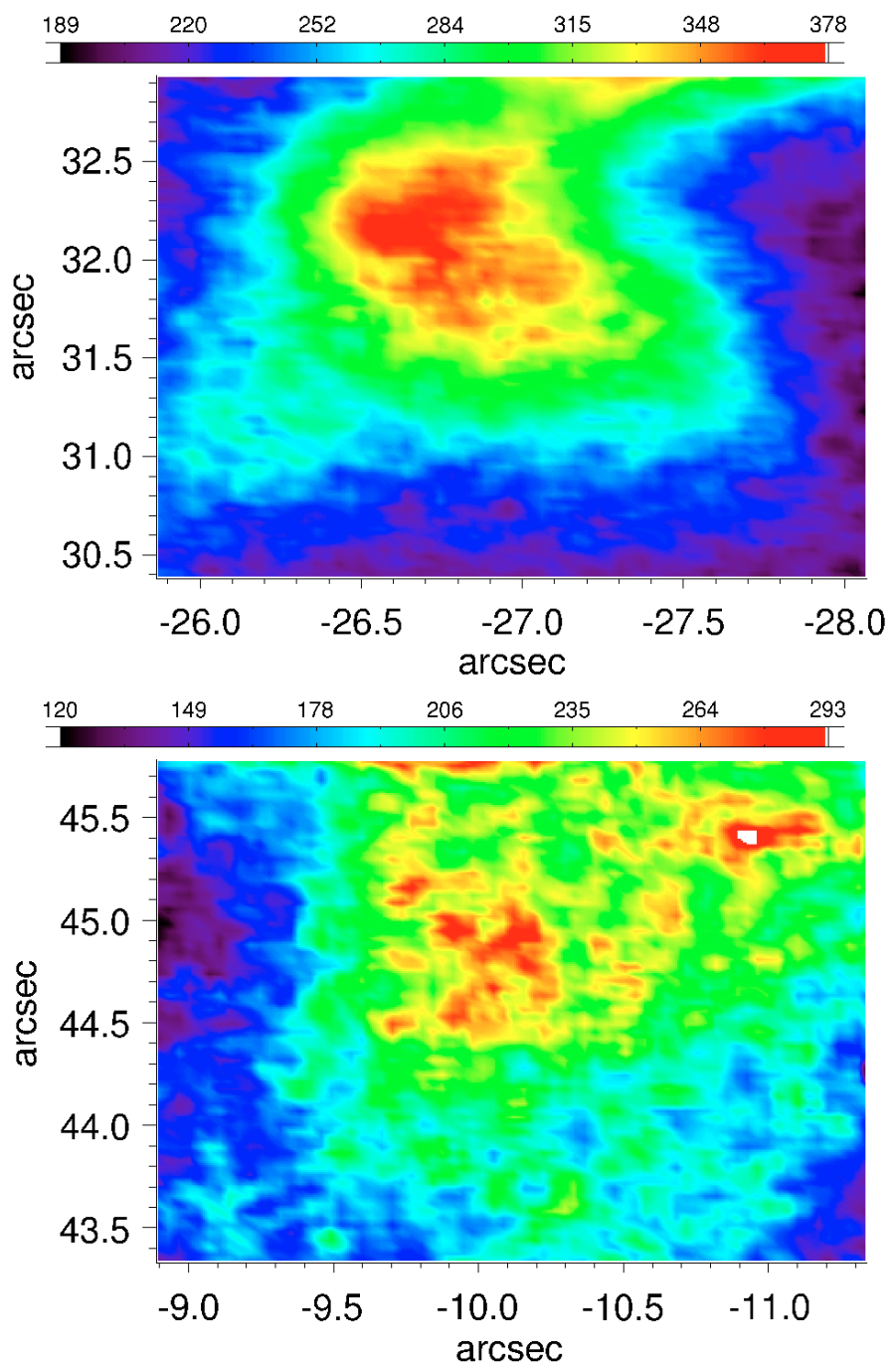

Fig. 9. a) Flow No. 1-57: see Table A.1. A smoothly structured WRV situated in Peak 1 but not in the flow front described in the text: Sect. 4.3. b) Flow No. 1-7: see Table A.1. A highly structured WRV feature lying in the flow front. The bar above shows brightness in counts per $400 \mathrm{~s}$.

data but showing the morphology in graphic detail, and the right hand side showing the GriF results, including velocity data as contours.

The structure in region B appears at first to consist of disconnected fragments of emission, which may reflect inherent clumpiness in the gas. However, on close examination it is found that there is weak emission connecting some of the prominent bright features shown in Fig. 4. An example is shown in Fig. 12. A ridge of very weak emission, at the level of 30-60 counts per $400 \mathrm{~s}$, corresponding to velocity uncertainties of $5-8 \mathrm{~km} \mathrm{~s}^{-1}$ $(3 \sigma)$, can be identified connecting three bow-shocks. Velocities in this ridge vary between -10 and $-30 \mathrm{~km} \mathrm{~s}^{-1}$. Thus the outflow region represents a concerted bulk motion, though diffuse in places.

\section{Discussion}

Discussion here centres on whether all the emission observed is due to action of outflows originating in massive stars buried in $\mathrm{OMC} 1$ or whether some emission is generated due to local events. Local activity could arise either indirectly due to the energy input from massive stellar outflows, causing a cascade of supersonic turbulence (Gustafsson et al. 2006a,b), or could arise from outflows from protostars actively forming within OMC1.

Turbulent motion or local outflows from low mass star formation would be expected to show no overall preferential direction of motion within the plane of the sky, with each site or group of sites acting independently of any other. This is in contrast to shocks associated with the large scale outflows which would be expected to inherit, at least in part, the direction of motion of the parent outflow. This point is examined in Sect. 5.1 below.

\subsection{The orientation of shocks in the plane of the sky}

This section considers how data for the displacement of the maxima in velocity and brightness in any chosen feature, recorded in the final column of Tables A.1-A.3, may be used for the determination of the orientation of shocks in the plane of the sky.

In brief a shock consists of two shock fronts: an outer bow shock which heats and accelerates the surrounding medium and an inner shock, the Mach disk, where the inflowing material driving the shock impinges on the material decelerated behind the outer shock. Relative to the surrounding gas we expect to see the highest velocity at the Mach disk, whereas the brightness is expected to peak at the apex of the outer shock.

On this basis the brightest emission and the highest velocity are expected to be closely spatially associated and the shock travels in a direction from maximum velocity to maximum brightness. This model will be referred to as the velocity to brightness model. In some cases observations clearly support this model. An example is shown in Fig. 11, object B-43, Table A.3. Here the maximum velocity is spatially displaced from the maximum brightness by $0.3^{\prime \prime}$ and the object gives every appearance of a bow shock travelling in the direction of maximum velocity to maximum brightness. A counter-example to the above is however furnished by object 2-20 in Table A.2. Here the bow shape of the emission suggests motion in a NW direction, whereas application of the velocity to brightness model for the shock motion indicates motion in the SE direction. Furthermore, in areas with a high spatial density of flows, perhaps indicative of a multiple star forming region (see Sect. 5.2), closely situated pairs of outflows where one is red-shifted and the other blueshifted are seen. However, for all pairs of red- and blue-shifted shocks where position angles are determined by the maximum velocity to maximum brightness flow direction model (see below), the flows appear to be converging in the plane of the sky. If any pair of flows formed a bipolar outflow, they would clearly be expected to be found diverging, that is, moving apart from one another. An example of this discrepant behaviour may be found in Fig. 17a showing flows 1-76 and 1-81 (see Table A.1).

Failure of the velocity to brightness model will arise if effects of optical depth are paramount in determining the location of maximum brightness. The brightness of emission is a function not only of the local efficiency of excitation associated with local loss of kinetic energy, but also of the column density of gas which is observed. Thus a shock travelling at some arbitrary angle may appear brighter behind the position of maximum velocity by virtue of its bow form, although locally the emission is weaker than at the tip of the shock, where the latter is of low column density. This model is illustrated in Fig. 13, and was described in G2003. This model will be referred to as the column density model.

The common feature of both models is that the maximum in emission and the maximum in velocity lie on a vector defining the orientation of the shock in the plane of the sky. However, in the absence of a full 2D or 3D C-type shock code for 


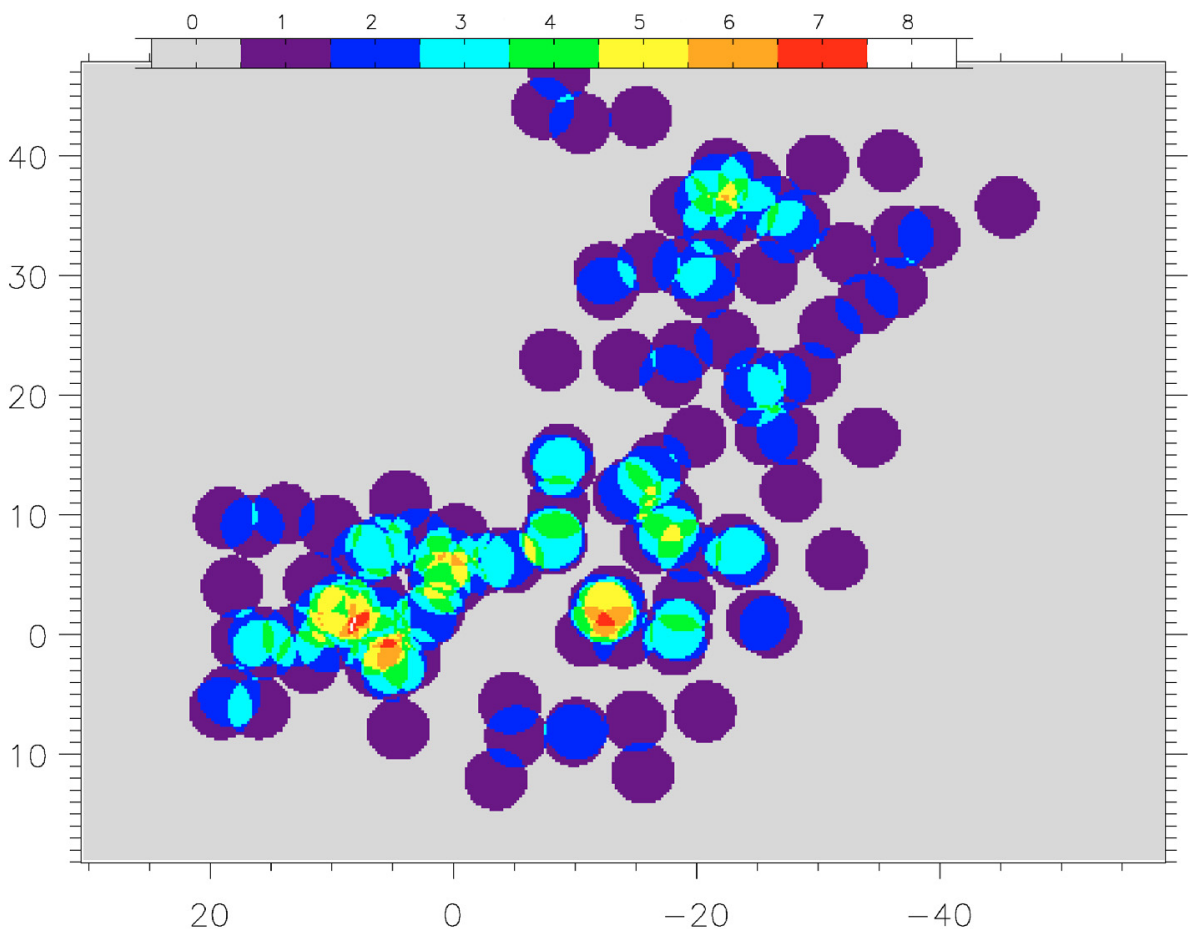

Fig. 10. Spatial density of emission features associated with flows, showing the number of flows within $\sim 1200$ AU of any given point. The colour scale illustrates the number of overlapping features at any point. See Sects. 4.4 and 5.2. Axes are labelled in arcseconds.
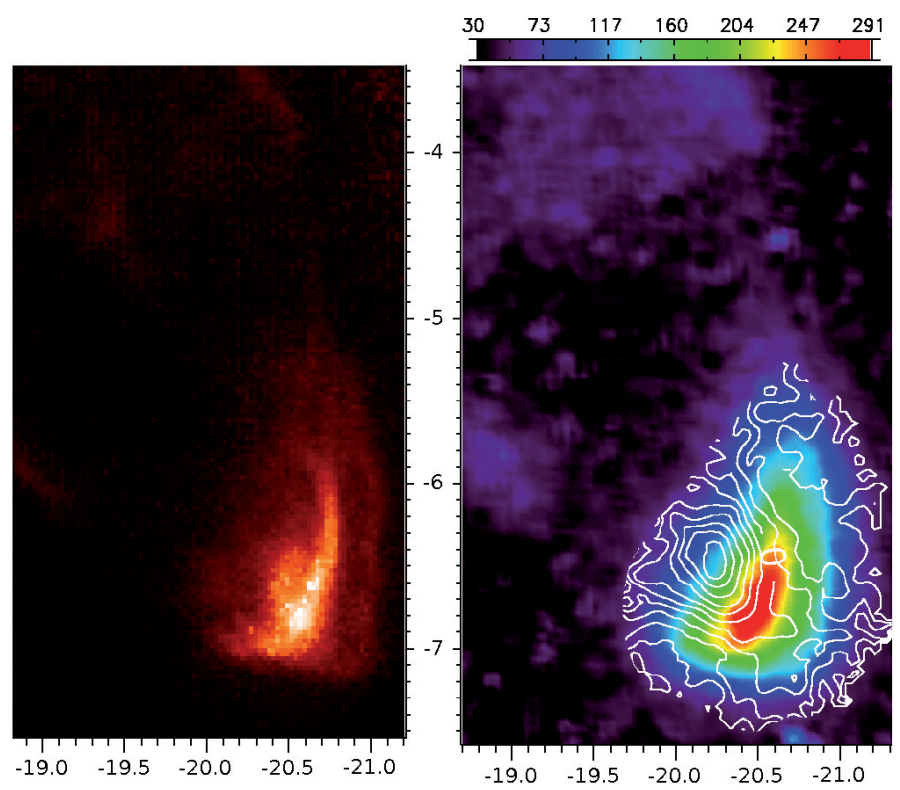

Fig. 11. Left hand panel: a $\mathrm{H}_{2} v=1-0 \mathrm{~S}(1)$ VLT image of flow No. B43, Table A.3, taken with the NAOS-CONICA adaptive optics system, with a spatial resolution of $~ 35$ AU: see Lacombe et al. (2004). Right hand panel: the same object obtained with the GriF instrument on CFHT, with a spatial resolution of 70 AU. Contours show velocity and colours show brightness in counts per $400 \mathrm{~s}$. The maximum in velocity is $-36 \mathrm{~km} \mathrm{~s}^{-1}$ at $-20.2^{\prime \prime} \mathrm{E},-6.5^{\prime \prime} \mathrm{N}$. The interval between contour lines is $4 \mathrm{~km} \mathrm{~s}^{-1}$.

$\mathrm{H}_{2}$ excitation including chemistry, it is not possible to determine which processes and thus which model may be relevant in any particular case, save where the morphology clearly indicates a bow shock (as in Fig. 11). Tables A.1-A.3 quote position angles measured east of north (i.e. counter-clockwise) based on the velocity to brightness model where shocks are travelling in the direction from the position of maximum velocity to the position of maximum brightness in the plane of the sky. However, with a few exceptions where the morphology of the flow would appear to dictate the absolute position angle, e.g. Fig. 11, these position angles are uncertain by $180^{\circ}$ with respect to absolute direction as discussed above. The exceptions are marked with a star and are found only in Table A.3 for the outflow region B. The uncertainty in the position angle is estimated to be $\sim \pm 25^{\circ}$, decreasing with increasing displacement. The uncertainty stems from the determination of the positions of the maxima. A displacement of $<0.25^{\prime \prime}$ of maximum brightness and maximum velocity is close to the resolution limit, and small variations in displacement would have a large influence on derived position angles. Position angles are therefore only determined where the displacement is $\geq 0.25^{\prime \prime}$.

It should be noted here that clumpiness in the medium into which the shock is propagating could result in significant deviation between the vector joining the maximum in velocity and brightness for a given shock and the true velocity vector of the shock. In separate work in progress, we have compared the orientation angles found here with proper motion data (N. Cunningham, private communications). With the limited data presently available, we find that there appears to be good agreement (e.g. $\pm 30^{\circ}$ ) between the velocity vectors determined from proper motion studies and those derived on the basis described here.

An important consideration is whether the position angles are dominated by a general outflow motion from the BN-IRc2 area or are approximately isotropic in distribution in each main region (Peak 1, Peak 2 and region B). Figure 14 show the distribution of position angles binned over $30^{\circ}$ intervals, with the reservation that individual position angles may be incorrect by $180^{\circ}$ (see above discussion). In this connection, the assumption has been made that one or the other model for position angles (velocity to brightness model or column density model) will dominate in a given zone. On this assumption, any preferred directions can be found by looking for anisotropies in the distribution of position angles. Rebinning the histograms in Fig. 14 to just two bins of width $180^{\circ}$ and then shifting the absolute positions of the bins to cover all possible combinations will show 


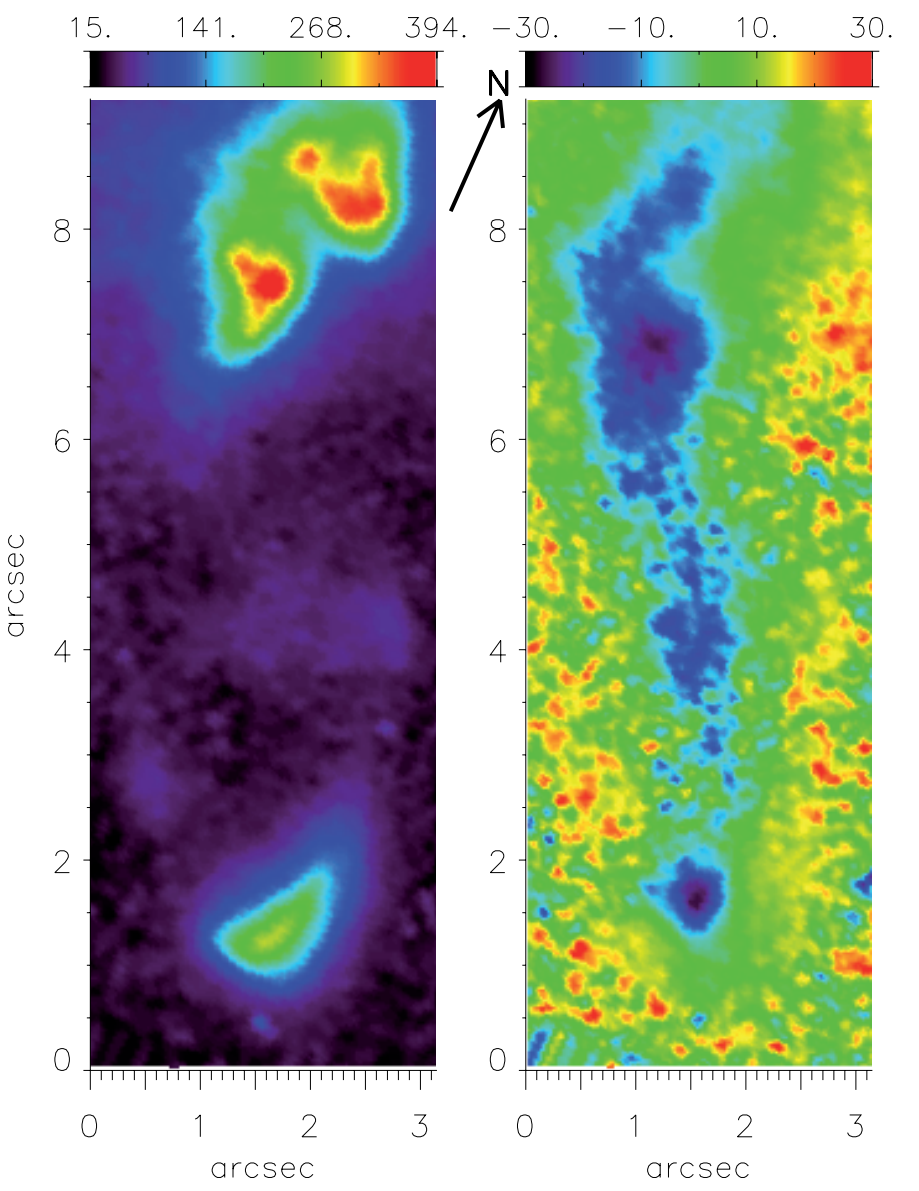

Fig. 12. GriF data for three bow shocks within the outflow region B. Left-hand image: $\mathrm{H}_{2}$ emission brightness in $v=1-0 \mathrm{~S}(1)$. The bar above shows brightness in counts per $400 \mathrm{~s}$. Right hand image: velocity data for the same field. The colour scale show velocity in $\mathrm{km} \mathrm{s}^{-1}$. The orientation of the images is such that north points at an angle of $24^{\circ}$ measured clockwise as indicated on the figure. The objects involved, lying $15^{\prime \prime}$ to $20^{\prime \prime} \mathrm{SW}$ of source I (see Fig. 4) are B-43 to the south (see also Fig. 11) and B-35-37 in the north, see Table A.3. Axes are relative to the bottom left hand corner of the figure, not to TCC0016.

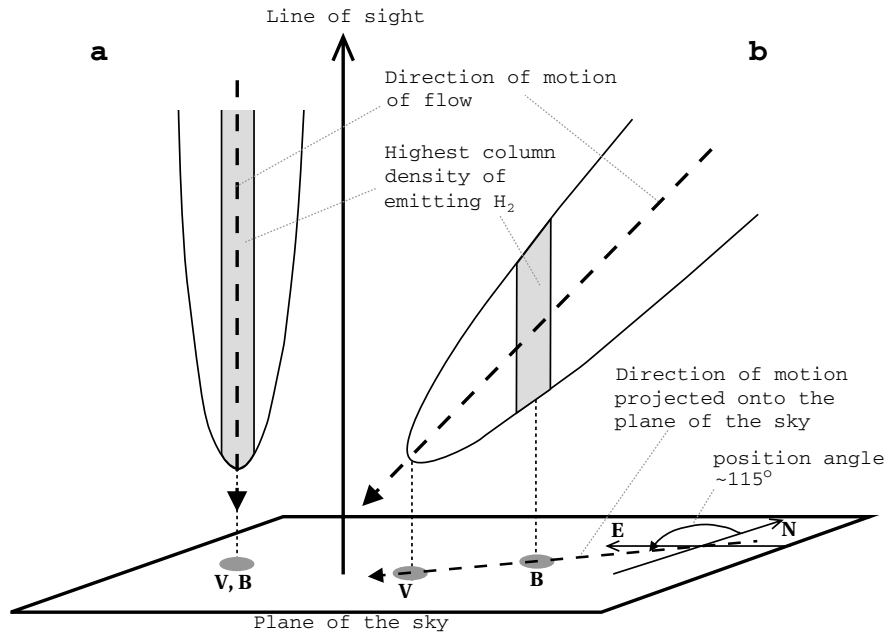

Fig. 13. An illustration of the proposed geometry by which position angles are estimated in the column density model (see Sect. 5.1). V's marks the position of the observed maximum in velocity and B's in brightness in the plane of the sky. a) A flow moving in the line of sight: the maxima in brightness and velocity coincide. b) A flow moving at an angle to the line of sight: the maximum in brightness will appear at some point behind the tip of the shock. A vector through the two maxima then gives the direction of the flow projected onto the plane of the sky, the position angle.
Table 1. Good protostellar candidates, "candidates", (upper half of table) and weaker candidates, "possible candidates", (lower half) in the area of OMC1 covered in the present work, based on data taken from Muench et al. (2002). For purposes of cross-reference, we have adopted the numbering of Muench et al. (2002).

\begin{tabular}{ccccc}
\hline \hline \multicolumn{5}{c}{ Protostellar candidates in OMC1 } \\
\hline \multicolumn{5}{c}{ Candidates } \\
$K-L>1.5 ;$ & $J-H>1.0$ or $J-H$ undetermined. \\
\hline Muench et al. & Coordinates & Colour indices \\
numeration & rel. to TCC0016 & $K-L$ & $J-H$ \\
\hline 00506 & -18.3 & -17.1 & 5.77 & - \\
00543 & 9.5 & -7.2 & 3.29 & - \\
00557 & -0.9 & -5.0 & 2.32 & $>1.82$ \\
00560 & -38.4 & -2.3 & 3.03 & $>0.82^{a}$ \\
00569 & -24.0 & -0.1 & 3.57 & - \\
$00572 \mathrm{~A}$ & -3.6 & 0.6 & 1.52 & $>2.67^{a}$ \\
00576 & 19.2 & 1.6 & 1.77 & 1.07 \\
00578 & -14.6 & 1.6 & 4.42 & - \\
00580 & 4.4 & 2.4 & 2.76 & 3.77 \\
00583 & -8.3 & 3.0 & 1.50 & 1.86 \\
00596 & 14.6 & 5.9 & 1.53 & 1.11 \\
00598 & -8.4 & 6.3 & 2.99 & 3.30 \\
00603 & -0.6 & 7.5 & 2.22 & 1.02 \\
$00614 \mathrm{~A}$ & -2.9 & 9.4 & 2.81 & $>4.65^{a}$ \\
00645 & -17.6 & 17.1 & 1.82 & 1.32 \\
$00709 \mathrm{~A}$ & -9.2 & 34.7 & 1.99 & 1.25 \\
00726 & -16.7 & 39.4 & 2.55 & 2.94 \\
\hline \multicolumn{5}{c}{ Possible candidates } \\
No $L$ band $; H-K>2.0$ or $K$ band photometry only. \\
\hline Muench et al. & Coordinates & \\
numeration & rel. to TCC0016 & $H-K$ & $M_{K}$ \\
\hline 00552 & -20.9 & -6.1 & 2.81 & 13.03 \\
00554 & -4.7 & -5.8 & $>2.79$ & 15.01 \\
00561 & 15.8 & -2.1 & 3.43 & 13.22 \\
00570 & -5.7 & 0.0 & $>3.00^{b}$ & 14.80 \\
00585 & -10.7 & 3.4 & 3.19 & 10.96 \\
00591 & -11.1 & 4.9 & 3.49 & 10.97 \\
00594 & 1.5 & 5.2 & $>3.85^{b}$ & 13.95 \\
00609 & -40.8 & 8.3 & $>1.63^{b}$ & 16.17 \\
00610 & -9.2 & 8.5 & 6.09 & 12.44 \\
00634 & -8.6 & 14.2 & 3.35 & 10.78 \\
00665 & -17.1 & 21.7 & 2.04 & 11.01 \\
00691 & -25.1 & 29.3 & 3.05 & 13.39 \\
00695 & -37.4 & 30.4 & 3.13 & 15.59 \\
00698 & -44.0 & 31.1 & $>2.91^{b}$ & 14.89 \\
00713 & -47.7 & 36.1 & $>2.99^{b}$ & 14.81 \\
\hline
\end{tabular}

${ }^{a}$ Muench et al. quote a detection limit of 18.15 in $J$ band. ${ }^{b}$ Muench et al. quote a detection limit of 17.8 in $H$ band.

anisotropies as large differences in the number of flows in each bin. This corresponds to placing a line across the star diagrams in the inset in Fig. 4 and counting the number of flows on each side of the line. If the line is rotated (corresponding to the bins in Fig. 14 being shifted) any preferred direction will appear as a maximum in the ratio between the number of flows on each side of the line when it is at an angle perpendicular to the outflow direction, decreasing to roughly unity as the line is rotated to be parallel with the direction of overall motion. If there is no preferred direction of motion the ratio should be approximately unity independent of the angle of rotation. Figure 15 shows this ratio as a function of angle measured counter-clockwise from north for each main region. The ratio is calculated so that it is always $\geq 1$.

Region B (Fig. 15c) shows a high degree of anisotropy around $140^{\circ}-160^{\circ}$ and a minimum around $50^{\circ}-70^{\circ}$. As 

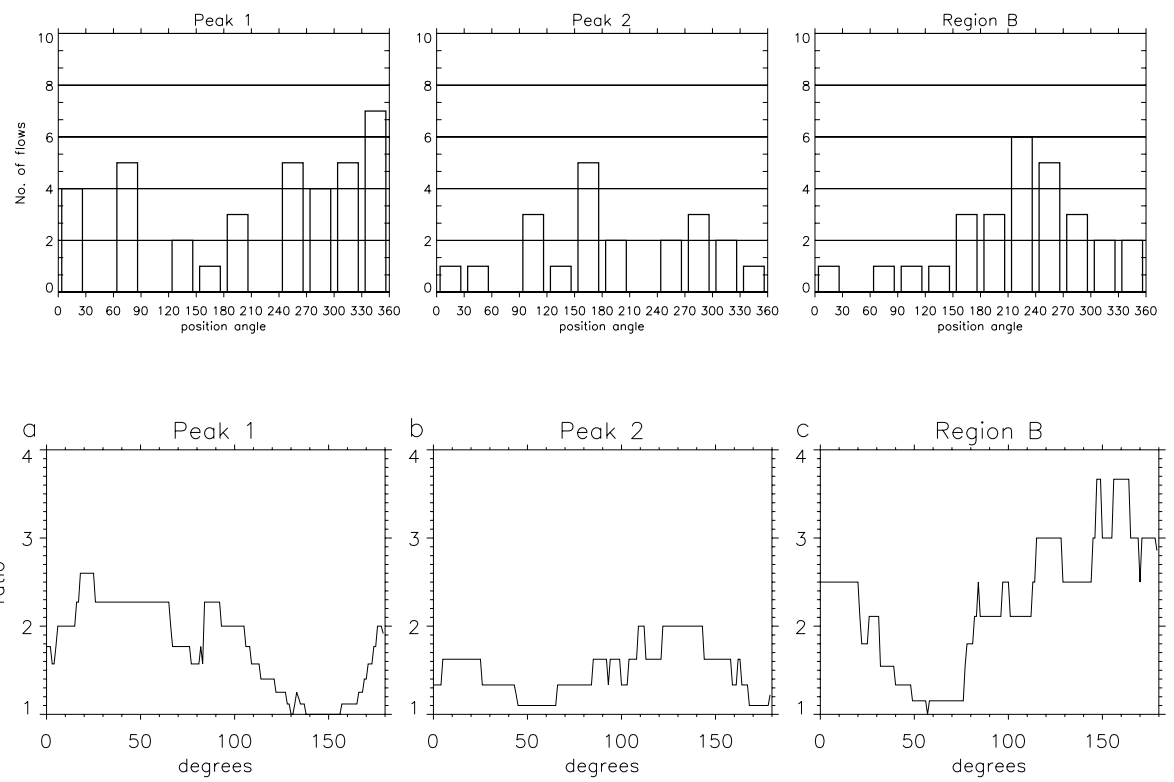

Fig. 14. Number of flows in each of the three main regions with position angles in a given range (see also star diagrams in Fig. 4).

Fig. 15. The ratio between the number of flows on each side of a line through the star diagrams in Fig. 4 as a function of the position angle of the line. A high value suggests an anisotropy in the distribution of position angles across the line while a value of unity indicates an isotropic distribution. described above, this property, with a maximum and a minimum perpendicular to the maximum, indicates that the motions are dominated by an overall outflow motion. In this case the motion is along a line oriented at position angle $\sim 50^{\circ}-70^{\circ}$, that is, approximately NE-SW. The nature of this outflow in region B will be discussed further in Sect. 6 .

Figure $15 \mathrm{a}$ (for Peak 1 ) shows a minimum around $130^{\circ}-160^{\circ}$ and a maximum at $20^{\circ}-50^{\circ}$. This is similar to the structure observed for region $\mathrm{B}$ although the maximum is less pronounced. This suggests that the observed flows in Peak 1 are also partly described by an outflow motion (towards the NW in this case), but not to the same degree as in region B. Finally Peak 2 (Fig. 15b) shows only very weak maximum-minimum structure, with a distribution of position angles which is close to isotropic. This in turn suggests that the flows observed in Peak 2 are only weakly affected by general outflow motion and tend rather to be individual flows, such as might arise from local star formation or turbulence.

\subsection{Protostars and star forming regions in OMC1}

Patterns of gas motion and spatial distribution of flows are examined here for Peaks 1 and 2. Our discussion provides arguments in favour of on-going low mass star formation in OMC1. The major source of energy injection into $\mathrm{OMC} 1$ is at $0.1 \mathrm{pc}$ scales (20000 AU) through outflows from massive stars and as part of a large scale cascade of turbulent energy (Gustafsson et al. $2006 \mathrm{~b})$. Thus the major proportion of the flows, both large and small scale, identified in the present work are an expression of the turbulence inherent in the gas through massive stellar outflows from the BN-IRc2 complex (Shuping et al. 2004; Greenhill et al. 2004a,b) and of shocks generated by the passage of ejecta such as the bullets. However the gas dynamics may also be influenced by low mass star formation. Outflows from low mass stars would provide an additional source of energy and generate flows at smaller scales of a few arcseconds, 1000-2000 AU, through bipolar outflows from isolated protostars or multipolar outflows from multiple star formation (see Figs. 17a-c). Such events correspond to the additional energy input on these scales that was detected in Gustafsson et al. (2006a,b). To examine this possibility we have made a search of the available literature to look for evidence for protostellar sources in OMC1. We have then looked for any spatial correlations between the shocks observed here and the positions of protostellar objects.

We first consider the IR observational evidence for the presence of early protostars in OMC1. To this end we have compiled a table of protostellar candidates in OMC1 (Table 1). We use the criterion, reported in Kenyon \& Hartmann (1995) for YSOs in Taurus, that an object with $K-L>1.5$ is almost always of a protostellar nature (specifically class I) and assume that this also holds for Orion. We apply this criterion to the photometry data of Muench et al. (2002) and Lada et al. (2004) for Orion. We furthermore use the criterion that $J-H$ must be $>1.0$ to exclude, for example, unresolved binaries (Lada et al. 2000). The resulting protostellar candidates are shown in Table 1. For ease of reference we have adopted the numbering system of Muench et al. (2002). Where a ">" symbol is encountered, this implies that $J$ band data are absent, in the upper section of the table, or $H$-band are absent in the lower.

We have divided objects into those which are very likely protostars, labelled "candidates" (upper part of Table 1) and those where the identification is more tentative, labelled "possible candidates" (lower part of Table 1). In addition there are objects for which the colours do not suggest that they are protostellar in nature. These are not shown in Table 1. This last category forms the majority of point-like objects within our complete field. Within Peaks 1 and 2, these objects occur in roughly equal numbers to the sum of "candidates" and "possible candidates". Figure 16 shows the positions of all objects in all three categories. Candidates and possible candidates have been marked with circles and triangles respectively with their accompanying number for reference to Muench et al. (2002) and Table 1. Non-candidates have been marked with a square. From a comparison with Fig. 1 in Shuping et al. (2004) we find that among the objects in Table 1 are source n (00598), IRc4 (00585), IRc7 (00610), IRc11 (00614) IRc12 (00603) and IRc15 (00634). Furthermore IRc9 is found to be identical with 00726. Source $n$ is discussed in more detail in Sect. 6. IRc9 will be mentioned in the following section, Sect. 5.3.

The term "candidates" covers objects which have $K-L>1.5$ and which have either $J-H>1.0$ or where $J-H$ is undetermined. The term "possible candidates" covers objects with 


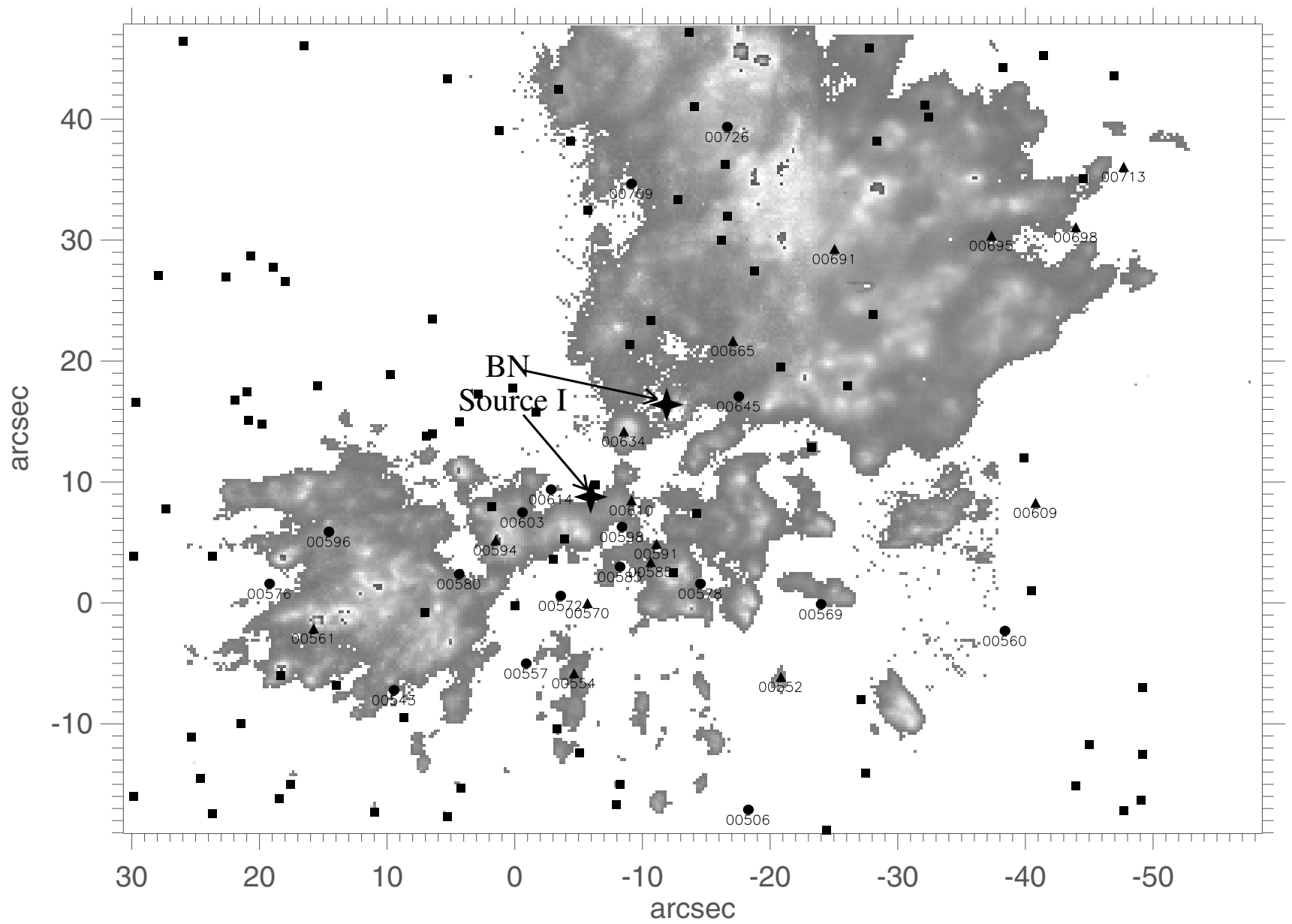

Fig. 16. The positions of point-like sources from Muench et al. (2002) in the observed field of OMC1. Objects have been divided into three categories based whether they are candidates (circles), possible candidates (triangles) or non-candidates (squares). Numbers refer to Muench et al. (2002) numeration (see Table 1). Note that a few objects to the far NE, SE and SW are outside our field of view but are included here for completeness.

$H-K>2.0$. As far as the authors are aware, the latter is a new criterion for the identification of protostars, motivated by a lack of $L$-band photometry. The new criterion has been established as follows. An extensive analysis of the $H-K$ colour index of the stars in Muench et al. (2002) has been carried out. It has been found that for objects with $K-L>1.5,39 \%$ have $H-K>1.5$ and $14 \%$ have $H-K>2$. By contrast, for objects with $K-L<1.5$ - which are not protostellar candidates - less than $2 \%$ have $H-K>1.5$ and only one object out of 300 has $H-K>2$. On this basis a $H-K$ colour index of $>2$ is adopted as a tentative identification of "possible protostars" in Table 1 and in Fig. 16. We note that the value of $H-K$ cannot however be used to exclude protostellar candidates. In this sense the objects listed in Table 1 form a minimum set. Under "possible candidates" in Table 1 we also list object from Muench et al. (2002) which have only been detected in $K$ band. These are shown with lower limits for $H-K$. The $K$-band detection, without $J$ and $H$, suggests that these objects are deeply buried in the dusty gas. We conclude that there are 17 good protostellar candidates in our field with a further 15 possible candidates.

A brief comparison has also been made with the Chandra Orion Ultradeep Project (COUP) X-ray data (Getman et al. 2005; Grosso et al. 2005). COUP contains numerous point sources in the direction of the region studied here. About half of these are found to be obscured by column densities of $>10^{22} \mathrm{~cm}^{-2}$ (Grosso et al. 2005). A comparison of the sources in Table 1 with the COUP data in Grosso et al. (2005) shows a remarkable correspondence between candidates in Table 1 (upper part of table) and COUP sources with 14 out of 17 spatial overlaps. Most of these are rated as obscured, that is, $N_{\mathrm{H}}>10^{22} \mathrm{~cm}^{-2}$ in Grosso et al. (2005). The so-called possible candidates in table 1 (lower part of table) are anti-correlated, with only 3 out of 15 spatial overlaps with COUP data, where all 3 overlaps are with obscured COUP sources. Since early protostellar objects (e.g. class I) may commonly be X-ray emitters (Koyama et al. 1996; Feigelson et al. 2003), this comparison with COUP data provides additional evidence that some of the point sources buried in $\mathrm{OMC} 1$ may be protostellar in nature.

We now turn to the positional correlation of protostellar candidates with possible outflows and $\mathrm{H}_{2}$ emission in general. In some regions two closely spaced shocks, where one is redshifted and the other blue-shifted, are seen moving in opposite directions. This could indicate that a protostar is situated in between, causing the shocks through a bipolar outflow. An example of this is shown in Fig. 17a, where such a pair of flows are found. Data in Table A.1 show that position angles of these two flows are roughly $180^{\circ}$ apart, noting the indeterminacy with regard to their relative direction. If the positions of protostellar 


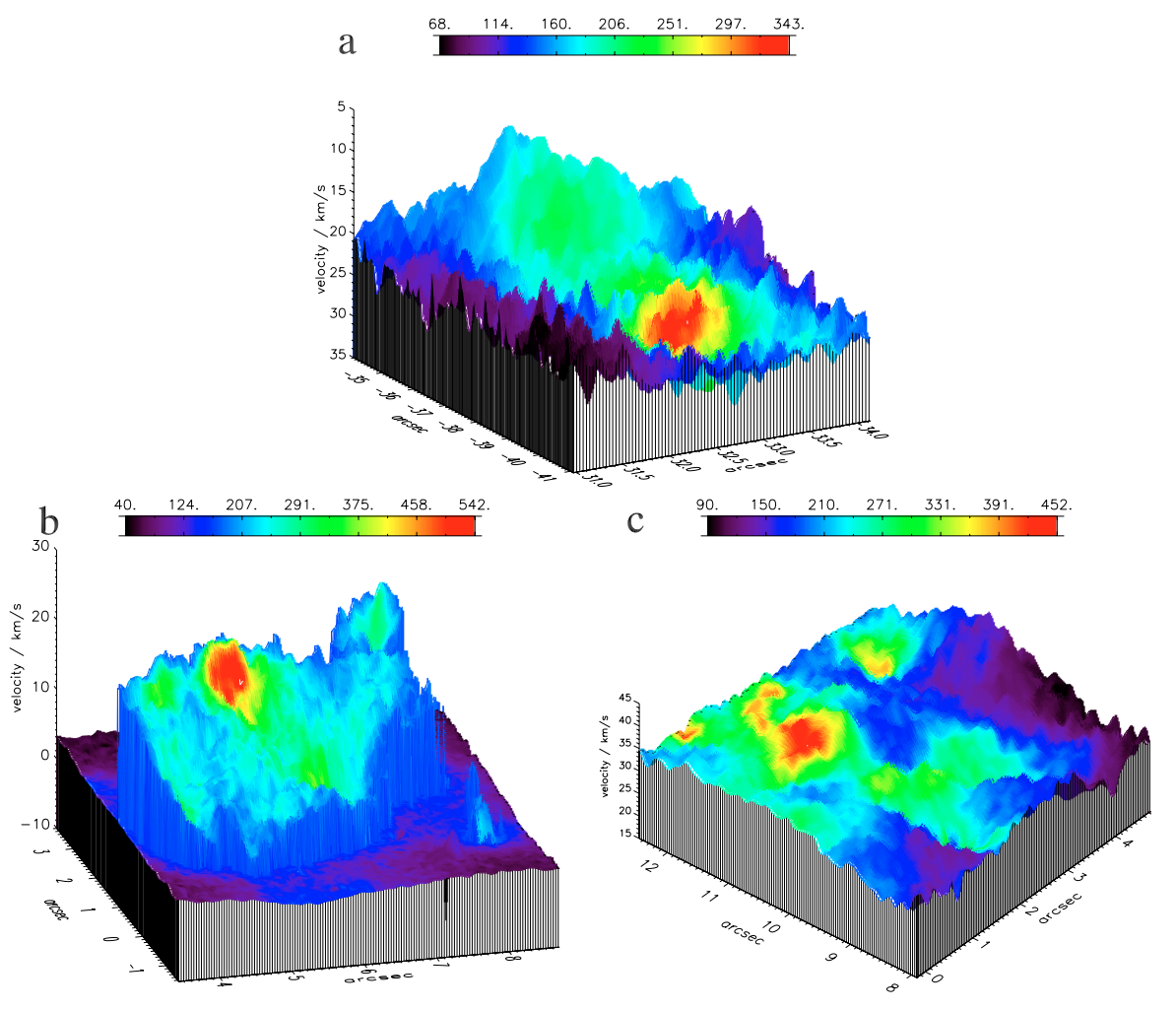

Fig. 17. Various areas with multiple flows in a small area: a) a possible bipolar outflow. Flows No. 1-76 and 1-81: see Table A.1. b) A complex flow pattern. Flows Nos. 2-42, 45, 46, 47, 48, 49: see Table A.2. c) Another example of a complex flow pattern. Flows Nos. 2-16, 18, 19, 20, 24, 25: see Table A.2. The bar above shows brightness in counts per $400 \mathrm{~s}$. candidates in Table 1 are compared with the positions of candidate bipolar outflows, there is no clear correlation between the two. In only 2 positions, $15.75^{\prime \prime} \mathrm{E}-2.1^{\prime \prime} \mathrm{N}$ (object 00561 , see Table 1) and 1.50" E 5.2" N (00594) a candidate protostar appears directly over such a pair of flows (these are marked with " $\dagger$ " in Table A.2), whereas in many cases a "bipolar pair" is found where there is no evidence of a protostar. Thus a "bipolar pair" in itself does not appear to be evidence of the presence of a protostar. However caution should be exercised since we are considering the earliest stages of protostellar development (class $0 / \mathrm{I}$ ). This phase is characterized by a very high degree of obscuration, and new, deeper observations than those of Muench et al. (2002) used here to detect protostars may yield a different conclusion.

As described in Sect. 4.4, there is a marked difference in how flows are distributed in Peak 1 and Peak 2. In Peak 1 the flows are mostly uniformly distributed across the area, whilst in Peak 2 shocks often appear in small groups of 6-8 shocks within an area of $\sim 5^{\prime \prime}$ in diameter (see Figs. 10 and $17 \mathrm{~b}, \mathrm{c}$ ), which corresponds to $\sim 2400 \mathrm{AU}$ at the distance of Orion. Since most stars are expected to form as part of a binary or multiple system (Pudritz 2002; Larson 2003), it seems plausible that these groups of shocks may indicate places where multiple star formation is ongoing: the presence of 2-3 protostars may account for several shocks from outflows. The flows in these regions are not however generally found as pairs of shocks moving in opposite directions, as may be seen in Figs. 17b and c with reference to the position angles in Table A.2. This would support a picture in which multiple star formation is a complex process involving interactions between the protostars and disks causing jets which are episodic or pulsed (Larson 2003, and references therein). These multiple shock features may alternatively reflect a region of especially active turbulence or may also be explained by, say, a bullet passing through an area containing small clumps of dense gas, resulting in several smaller shocks propagating into the local medium.
In the following we examine whether we can associate regions with large densities of flows, as just mentioned, with protostars listed in Table 1. Moving from east to west (left to right) in Fig. 10 the first region of high flow density is around $7^{\prime \prime} \mathrm{E} 0^{\prime \prime} \mathrm{N}$. Only one stellar-like object is observed along the line-of-sight to this region: the proplyd 154-240 (7.4" E, -0.9" N) (O'Dell et al. 1997; Schultz et al. 1999). Since this object has been observed in the visible by HST it cannot be a protostar buried in the dense, dusty gas.

Moving away from a line-of-sight positional correlation of possible protostars and $\mathrm{H}_{2}$ emission, there is a protostellar candidate $\sim 4^{\prime \prime}$ NW or 1600-2000 AU in the plane of the sky. This is the object 00580 (Table 1 ) which has $K-L, J-H>2$ fulfilling our criteria for a buried protostar. This is therefore a good candidate to power at least some of the emission that is observed in this highly active zone.

The second region of high flow activity in Fig. 10 is around coordinates $0^{\prime \prime} \mathrm{E}, 6^{\prime \prime} \mathrm{N}$. Within a radius of a few arcseconds there is one object which satisfies the protostellar criteria, namely 00603 with $K-L=2.22$ and $J-H=1.02$. There are two other objects for which we do not have $L$ band data available and therefore we are unsure of their nature. One of these is 00594 which is listed in Table 1 under "possible candidates". This object lies just outside the Lada et al. (2004) frame. Muench et al. (2002) do not detect this object in $J$ and $H$ and record $m_{K}=13.95$. A further object is 00606 . This object has no $L$-band data, and with $H-K=0.57$ this is not an obvious protostellar candidate. However, one should recall from the above discussion that the $H-K$ colour index is useful only for including, not excluding, protostellar candidates. In this connection, 00606 is listed as being obscured by a column density of $8 \times 10^{22} \mathrm{~cm}^{-2}$ in COUP data and therefore this object is an embedded source and thus a reasonable protostellar candidate.

We turn now to the third flow concentration, which is centred on $-22^{\prime \prime}$ E $37^{\prime \prime}$ N. Surveying the region within a radius of $\sim 4 "$, 
we find the star IRc9 (00726) (Smith \& Bally 2005), with $J-H$ and $K-L$ both $>2$, fulfilling the protostellar criteria, see Table 1 .

Looking at Peak 1 in general, rather than at concentrations of flows, there are a further two protostellar candidates, 00645 and 00709A, both situated along the edge of the $H_{2}$ emission seen in Peak 1 . There are also another 5 possible protostellar candidates (00665, 00691, 00695, 00698, 00713) which are spread across Peak 1. In Peak 2, there are a further 3 protostellar candidates (00543, 00576, 00596), and one possible candidate, 00561, associated with the very bright $H_{2}$ emission at $16^{\prime \prime} \mathrm{E}-2^{\prime \prime} \mathrm{N}$. We note that with the exception of 00561 (in Peak 2) no stars or protostars in Peak 1 or 2 are directly associated with the brightest areas of $\mathrm{H}_{2}$ emission.

In region $\mathrm{B}$ there are 8 protostellar candidates and a further 8 possible candidates. Half of these are situated in the dense core of OMC1 around Source I and may contribute to $\mathrm{H}_{2}$ emission in this region. The other half are widely distributed over region $\mathrm{B}$, with some coincidences with $\mathrm{H}_{2}$ emission, for example 00569, a candidate in Table 1, or 00552, a possible candidate in Table 1.

The above considerations ignored the presence of WRVs in Peaks 1 and 2. In Peak 2 WRVs form only $20 \%$ of the observed flows but in Peak 1, they form more than $50 \%$ of the flows. However analysis presented in Sect. 4.3, showing the organised nature of WRV flows in Peak 1, suggests that many WRVs might be part of a subset of flows which are not appropriate for inclusion in the present analysis. The WRVs are discussed further in the following section, Sect. 5.3.

At the beginning of this section the question was posed of whether $\mathrm{H}_{2}$ emission might in part be caused by the presence of protostars with associated outflows. In brief the answer is that some small proportion of the emission is very likely caused by outflows from low mass stars. We have seen above that a number of protostellar candidates of class I are found in this area. As class I objects still have significant outflow activity this suggests that protostellar outflows may power some of the observed emission as suggested in Gustafsson et al. (2006b). There is not however a strong correlation between the location of shocks in our data and protostellar candidates. This suggests that most of the bright emission observed is due to large scale outflows and associated turbulent motion, as we also concluded earlier in Sect. 4.1. In particular in Peak 1, present data suggest that emission is dominated by massive outflow from the vicinity of Source I and the BN/IRc2 region. Because of the crowded nature of the $\mathrm{H}_{2}$ emission, it is difficult to ascribe specific flows to protostars, a difficulty compounded by obscuration. One exception to this is the area around a position $0^{\prime \prime} \mathrm{E}, 6^{\prime \prime} \mathrm{N}$ where we observe a small area with a high concentration of shocks and a number of protostellar candidates in and around the same area, suggesting that this may be a place of ongoing, multiple star formation.

\subsection{The origin of weak radial velocity structure in Peak 1}

We have seen in Sect. 4.3 that the spatial distribution of weak radial velocity components (WRVs) in Peak 1 revealed a band, $\sim 2-3^{\prime \prime}$ in width and more than $30^{\prime \prime}$ long, passing diagonally across emission in Peak 1 . It was also noted that $2 / 3$ of these WRVs showed a much more broken-up structure than other features, including other WRVs. This section briefly explores possible scenarios for the origin of this band of WRVs.

The first scenario is that the band of WRVs may represent a less energetic part of the outburst which created the "bullets" or "fingers" (Allen \& Burton 1993). These are clumps of gas moving outwards in the plane of the sky at velocities of up to several hundred $\mathrm{km} \mathrm{s}^{-1}$ (Doi et al. 2002) and appear to have an origin in the area around BN-IRc2 (Salas et al. 1999; O'Dell 2001; Doi et al. 2002; Bally \& Zinnecker 2005). This scenario is based both on the position of the band of WRVs across Peak 1 between the finger system and the BN-IRc2 complex and on the fact that the band is oriented orthogonally to the general direction of the outflow. This suggests a slower moving, denser part of the explosive outflow, which caused the finger system, ploughing through Peak 1 . With a distance of $\sim 26^{\prime \prime}$ from the area around sources I and $\mathrm{n}$, this implies a speed of $60 \mathrm{~km} \mathrm{~s}^{-1}$ in the plane of the sky assuming the age to be $10^{3} \mathrm{yr}$ (Doi et al. 2002). A shock velocity of $60 \mathrm{~km} \mathrm{~s}^{-1}$ limits the pre-shock density to a few times $10^{4} \mathrm{~cm}^{-3}$ for the propagation of C-type shocks (Le Bourlot et al. 2002). Shock models (Wilgenbus et al. 2000) show that the post shock density is about one order of magnitude greater and the brightness in the $v=1-0 \mathrm{~S}(1)$ line will approximately match that observed which is the order of $10^{-5} \mathrm{~W} \mathrm{~m}^{-2} \mathrm{sr}^{-1}$. The typical mass of each WRV can be estimated to be $\sim 5 \times 10^{-5} M_{\odot}$, given a post shock density of $3 \times 10^{5} \mathrm{~cm}^{-3}$ and an average diameter of $500 \mathrm{AU}$. This yields a total bulk energy for outflowing hot $H_{2}$ in the WRVs of the order of $10^{44} \mathrm{ergs}$, for the $47 \mathrm{WRVs}$ in Peak 1 . This is about $0.2 \%$ of the energy associated with the bullets (Burton 1997).

A second scenario is that the band of WRVs is the result of bright photodissociation zones powered by $\theta^{1}$ Ori $C$, but with enhanced brightness due to geometrical effects (see below). The morphology of the ionization front, described in Wen \& O'Dell (1995) and refined in O'Dell (2001), shows that the bright $\mathrm{H}_{2}$ clumps observed in the present work arise from dense gas within the H II region created by $\theta^{1}$ Ori C (Kristensen et al. 2003). Ionizing radiation has cleared away the diffuse gas and is now slowly eating its way through the denser clumps of gas remaining. Thus the line of WRVs along the eastern edge of Peak 1 may be the relic of a denser region that existed in the original molecular cloud. Whilst the brightness of the $\mathrm{H}_{2}$ emission cannot be reconciled with a face-on PDR (see Sect. 3.2), a variety of 3D geometries may result in some PDRs being observed edge-on leading to an increase in observed brightness. PDR emission may also be enhanced locally through a geometry originally proposed in Field et al. (1994) in the case of NGC 2023. In this model, a choppy surface of $\mathrm{H}_{2}$ yields chance lines-of-sight with high column density. This is consistent with the broken up appearance of $2 / 3$ of the features in this zone, mentioned in Sect. 4.3.

A third possibility is that the band of WRVs may be associated with IRc9 (00726 in Table 1 and Fig. 16). IRc9 is located $\sim 2^{\prime \prime}$ from the centre of this band. Smith \& Bally (2005) show that IRc9 is a YSO possessing a clearly defined disk. The major axis of the disk is oriented at right-angles to the WRV band which suggests that WRVs in the band could represent a highly collimated outflow in the plane of the sky from IRc9.

Apart from the 15 WRVs in the band, there are a further 32 spread largely NW of the band structure. These remaining WRVs themselves represent an over-representation for objects moving in the plane of the sky based on the analysis in Sect. 4.3. Present data do not provide any clear origin for these objects.

\section{The outflow zone, region B}

\subsection{The geometry of the region: radio observations and the present data}

An outflow in region $B$ has previously been identified in radioobservations involving both proper motions and radial velocity 


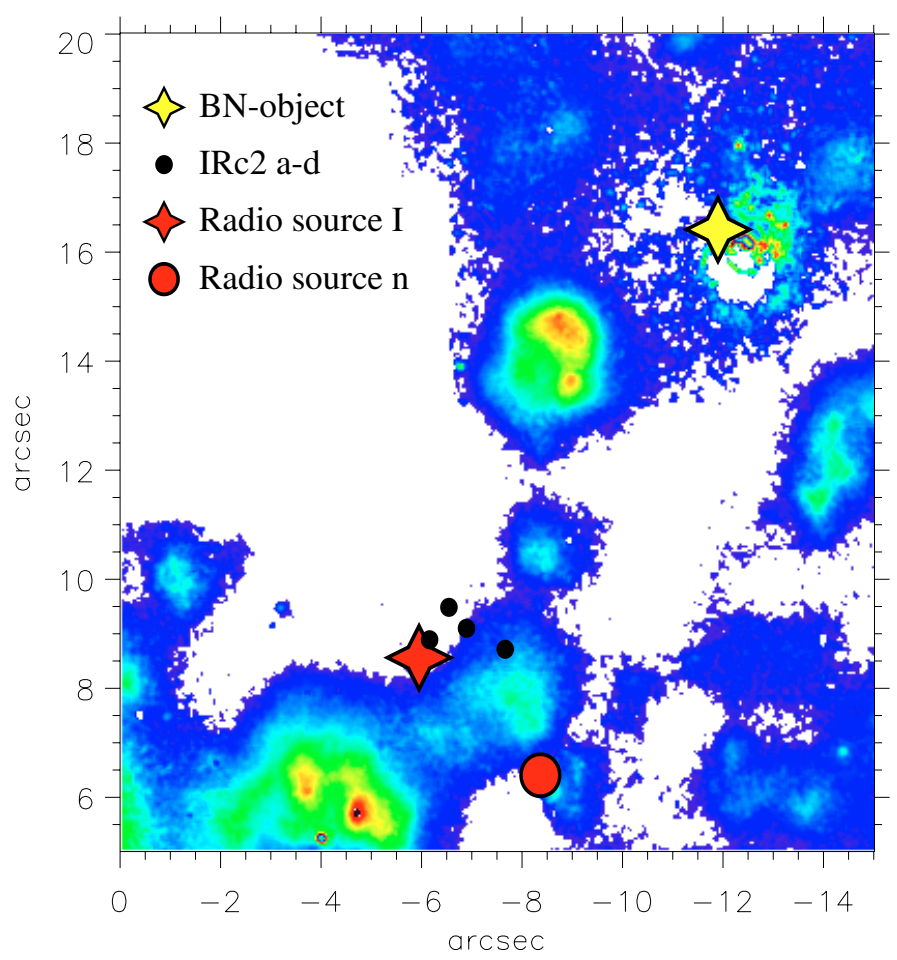

Fig. 18. The positions of $B N$, radio source $I$, radio source $n$ and IRc2 a$\mathrm{d}$, superimposed on velocity integrated emission in the $\mathrm{H}_{2} v=1-0 \mathrm{~S}(1)$ line.

measurements of $\mathrm{H}_{2} \mathrm{O}, \mathrm{OH}$ and $\mathrm{SiO}$ masers and thermal emission in $\mathrm{SiO}(v=0)$ and $\mathrm{NH}_{3}$, where data are reviewed in Genzel \& Stutzki (1989). Evidence of shocked gas within the flow is provided through observations of emission from high rotational states of $\mathrm{SO}, \mathrm{SO}_{2}$ and $\mathrm{HCN}$. The outflow has also been identified in subsequent observations reported in Wright et al. (1992, 1996) in data involving 16 molecular species, in particular SO and $\mathrm{SO}_{2}$ (Wright et al. 1996). The outflow originates from the vicinity of sources I and $\mathrm{n}$ whose locations are given in Fig. 18.

The expansion velocity associated with the above-mentioned outflow in region $\mathrm{B}$ on the basis of radio observations was found to be $18 \pm 2 \mathrm{~km} \mathrm{~s}^{-1}$ (Genzel et al. 1981). The data of Genzel et al. (1981) showed a flow extending $\sim 20^{\prime \prime} \mathrm{SW}$ of the BN-IRc2 region, which is very similar to the SW extent of the $\mathrm{H}_{2}$ emission recorded here. Genzel et al. (1981) also note, through their observations of numerous objects at closely similar velocity, that the flow appears to have been stable for $\gtrsim 3000$ years.

Our present observations measure gas motions relative to the ambient surrounding gas and therefore may also be interpreted as a radial component of an expansion velocity. The expansion velocity of $18 \pm 2 \mathrm{~km} \mathrm{~s}^{-1}$ derived from the observations of Genzel et al. (1981) matches accurately the mean velocity of $-18 \mathrm{~km} \mathrm{~s}^{-1}$ observed here for the hot $\mathrm{H}_{2}$ in region B: see Fig. 5. The velocities measured in this work are also consistent with data in Menten \& Reid (1995) and Wright et al. (1996).

On this basis we conclude that our observations show the IR counterpart of the so-called slow $\left(18 \mathrm{~km} \mathrm{~s}^{-1}\right)$ outflow in OMC1, hitherto only observed in the radio.

The mass outflow rate in region $\mathrm{B}$ may be estimated as follows. Typical gas densities in the $\mathrm{H}_{2}$ clumps in region $\mathrm{B}$ have been investigated in detail in Kristensen et al. (2006) and preshock values range between $10^{5}$ to $10^{6} \mathrm{~cm}^{-3}$, equivalent to an average density of $\sim 10^{-18} \mathrm{~g} \mathrm{~cm}^{-3}$. Assuming a continuous outflow and a measured velocity of $18 \mathrm{~km} \mathrm{~s}^{-1}$, this represents a mass flux of $2 \times 10^{-12} \mathrm{~g} \mathrm{~cm}^{-2} \mathrm{~s}^{-1}$. The total area in the plane of the sky covered by the excited $\mathrm{H}_{2}$ may be estimated by using a total area for each clump given by the area around each flow where the emission is $\geq 50 \%$ of the maximum brightness of the flow. The total area is found to be $\sim 5 \times 10^{33} \mathrm{~cm}^{2}$, giving a mass flow of $\sim 1.5 \times 10^{-4} M_{\odot} \mathrm{yr}^{-1}$. Including the red-shifted part of a bipolar outflow, this gives a total outflow rate of $\sim 3 \times 10^{-4} M_{\odot} \mathrm{yr}^{-1}$. Since only shock excited gas has been included this is a lower limit. This figure for the outflow rate is comparable to estimates given in Shepherd (2005) for stars of the luminosity associated with source I $\left(\lesssim 10 L_{\odot}\right)$. This estimate also agrees with the estimated mass loss of $10^{-2}-10^{-4} M_{\odot} \mathrm{yr}^{-1}$ given in Genzel et al. (1981).

The corresponding outflow momentum rate is $\sim 0.005 M_{\odot} \mathrm{yr}^{-1} \mathrm{~km} \mathrm{~s}^{-1}$ and mechanical luminosity is $\sim 8 L_{\odot}$. These values are typical of an early-type B-star (Shepherd 2005). The much debated issue of the origin of large scale outflows in OMC1, see for example Shuping et al. (2004), has recently achieved a new perspective with high spatial resolution (0.3-0.5") IR measurements in Shuping et al. (2004) and Greenhill et al. (2004a). These observations show that there are a number of potential YSO candidates buried within a zone in the north-east of region B. IRc2 was long believed to be the main power source in $\mathrm{OMCl}$ and the source of the outflow activity in the region. However these and other high resolution observations (Dougados et al. 1993) have resolved IRc2 into several individual sources. These may or may not be self-luminous (Shuping et al. 2004). At all events the current most likely candidates for outflow sources are sources I and n.

In the following we discuss the evidence presented in the literature for both source I and source $\mathrm{n}$ as the origin of the massive outflow in region B. Source I $\left(-5.95^{\prime \prime} \mathrm{E}, 8.56^{\prime \prime} \mathrm{N}\right)$, is a deeply buried O/B-star (Menten \& Reid 1995; Greenhill et al. 2004b). It is the most luminous source in the hot core of OMC1 with a luminosity of $5 \times 10^{3}$ to $10^{4} L_{\odot}$ (Menten \& Reid 1995; Beuther et al. 2004, 2006), compared for example to $\sim 2500 L_{\odot}$ for the BN-object (Gezari et al. 1998) or $\sim 2000 L_{\odot}$ estimated for source n (Greenhill et al. 2004a). Source I is obscured in the infrared and cannot be detected at wavelengths as long as $22 \mu \mathrm{m}$ (Greenhill et al. 2004a). Source $\mathrm{n}$ (object 00598 in Table 1, Greenhill et al. 2004a) is located at $-8.35^{\prime \prime} \mathrm{E}, 6.40^{\prime \prime} \mathrm{N}$ (Fig. 18).

\subsection{Source I as the origin of the region B outflow}

Based on their data for $\mathrm{SiO}$ and $\mathrm{H}_{2} \mathrm{O}$ emission around source $\mathrm{I}$, Greenhill et al. (2004b) proposed a model with an accretion disk and a wide angle outflow pointing NE-SW with an opening angle of $\sim 90^{\circ}$. This model replaces that of Greenhill et al. (1998) in which the axis of the outflow is rotated $90^{\circ}$ in the plane of the sky. The morphology of the outflow from source I proposed in Greenhill et al. (2004b), is shown in Fig. 19, lower left panel.

The axis of the outflow from source I passes through a region where we find a striking $\mathrm{H}_{2}$ emission feature. Velocity data for this zone are shown in the upper right hand frame of Fig. 19. Three fast shocks marked with arrows (flows No. B-7, B-9, B-10: see Table A.3) are seen impinging on the surrounding medium. A VLT image of the same area (Lacombe et al. 2004) is shown in the lower right-hand frame of Fig. 19. The model of the outflow from source I is superimposed upon it, to show the spatial relationship, where the accuracy of the relative positions is $\sim 0.2^{\prime \prime}$. The position of the maximum brightness of this outflow region, measured in $\mathrm{H}_{2}$, is $1.8^{\prime \prime} \mathrm{W}$ and $1.0^{\prime \prime} \mathrm{S}$ of source $\mathrm{I}$. The appearance of the $\mathrm{H}_{2}$ emission in this region suggests that this 

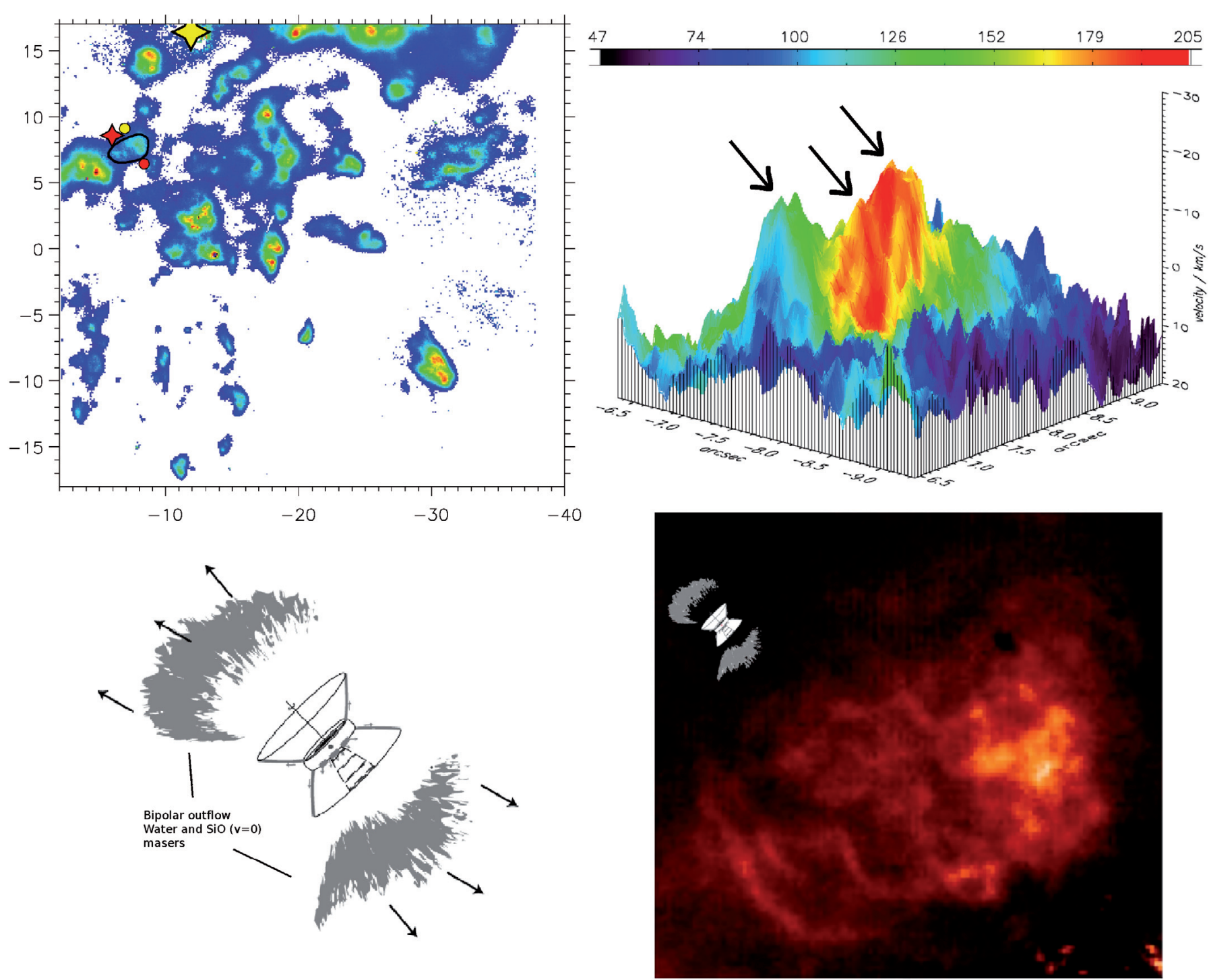

Fig. 19. Source I. Top left: region B recorded in the $v=1-0 \mathrm{~S}(1)$ line of $H_{2}$. The yellow star shows the position of BN and the red star shows the position of source I. The red circle is source $\mathrm{n}$, and the yellow circle is IRc2 $\mathrm{d}$ (compare to Fig. 18). The black outline shows the position of the $\mathrm{H}_{2}$ emission feature shown in the right hand panels. Bottom left: the geometry of the wide-angle outflow from source I taken from Greenhill et al. (2004b). Bottom right: VLT NACO image (3.2" by 2.9") from Lacombe et al. (2004) showing the outflow region (the "fireball") just SW of source I, outlined in black in the top left panel. The inset shows that the outflow from source I (not to scale) points directly at this region. Top right: velocity structure of the brightest part of the fireball, as seen from the $\mathrm{SW}$, showing three fast jets (arrows) impinging on the medium (flows No. B-7, B-9, B-10: see Table A.3).

is a zone where part of the outflow bursts through the dense gas surrounding source I. The morphology of this emission resembles the structure of shocks modelled in Blondin et al. (1989) and Lim et al. (2001) with the shocks seen face on.

The model of Greenhill et al. (2004b) of the outflow from source $\mathrm{I}$ is of a wide-angle bipolar outflow in the plane of the sky. This begs the question of why no $\mathrm{H}_{2}$ emission is observed as part of the outflow to the NE of source I. We would suggest that the outflow axis may in fact tip out of the plane of the sky with an inclination angle (to the line-of-sight) of $\lesssim 45^{\circ}$. This would explain the lack of $\mathrm{H}_{2}$ emission to the NE since emission would be rendered invisible through dust obscuration in this very dense zone (see below for an estimate of the IR extinction towards source I).

Our estimate of the inclination angle is based on three observations where our discussion is based on an opening angle of the outflow of $\sim 90^{\circ}$ (as suggested in Greenhill et al. (2004b), and typical of outflows from massive protostars, Shepherd 2005):

(i) In region $\mathrm{B}$ we see no red-shifted flows. This cannot be ascribed to obscuration, in contrast to the red-shifted lobe discussed above. A natural explanation for the lack of redshifted outflows in region $\mathrm{B}$ is that the flow has an inclination angle of $\lesssim 45^{\circ}$.

(ii) region $\mathrm{B}$ extends to the $\mathrm{NE}$ of source I. This can only arise if the inclination of the outflow cone is less than $45^{\circ}$. Note that we effectively observe the projection of this cone onto the plane of the sky. However region B only extends a few arcseconds north-east of source I, indicating that the inclination angle is close to $45^{\circ}$.

(iii) Greenhill et al. (2004b) observe a substantial overlap of $\mathrm{H}_{2} \mathrm{O}$ and $\mathrm{SiO}(v=0)$ masers along the line-of-sight. However these two molecular masers trace very different gas densities 


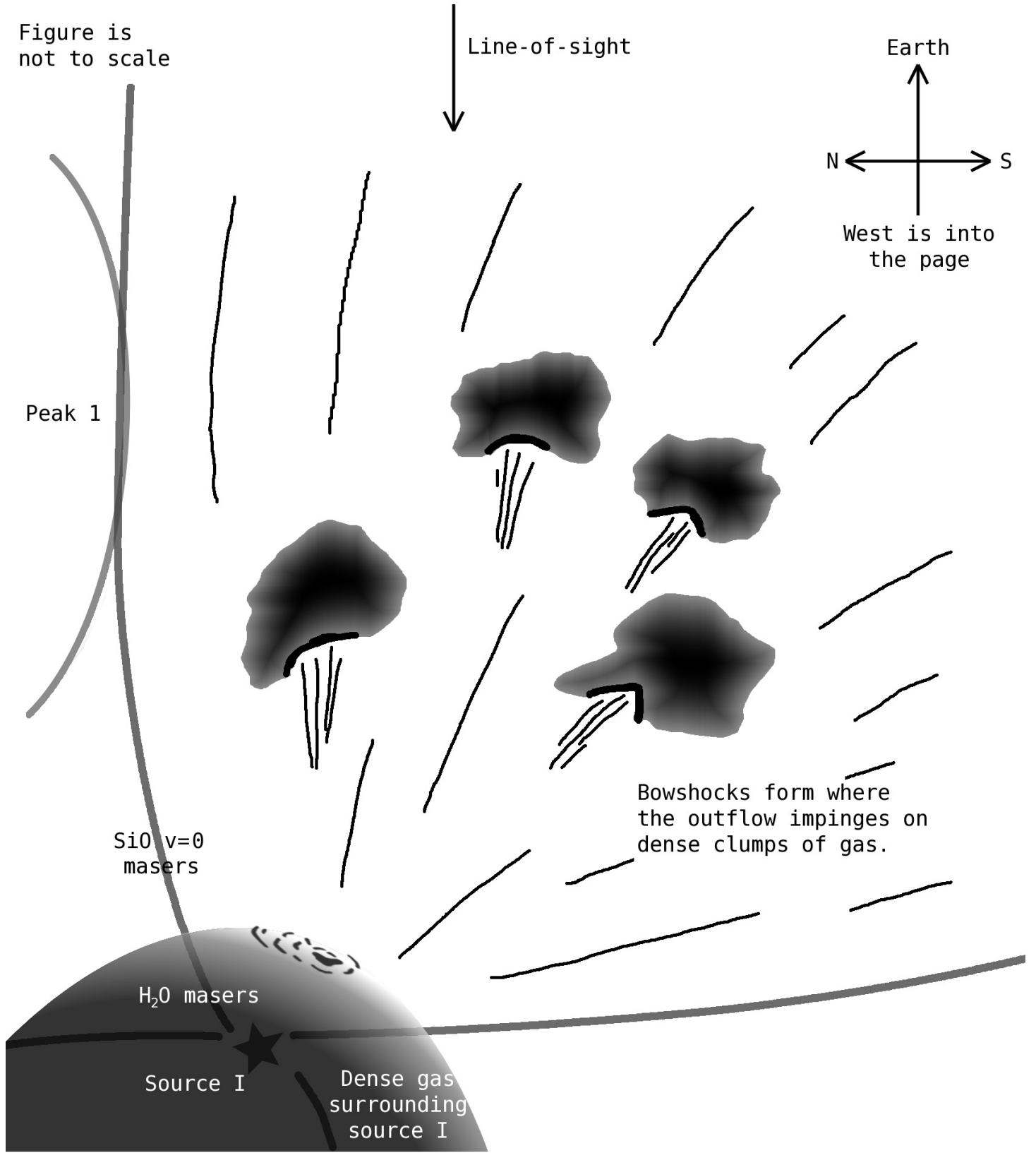

Fig. 20. The outflow from source $I$, showing the appearance of the outflow and its relationship to other objects in region B, seen at right-angles to the line-of-sight from a vantage point in Peak 2 looking towards the west.

and temperatures, and, as Greenhill et al. (2004b) note, should not be found within the same mass of gas. $\mathrm{H}_{2} \mathrm{O}$ masers may have associated kinetic temperature of 200 to $2000 \mathrm{~K}$, with a preference for temperatures around $600 \mathrm{~K}$, and densities of no more than a few $\times 10^{9} \mathrm{~cm}^{-3}$ (Yates et al. 1997). $\mathrm{SiO} v=0$ masers on the other hand trace gas densities of $10^{5-6} \mathrm{~cm}^{-3}$ (Greenhill et al. 2004b) and temperatures of 800-1600 K. If, as suggested here, the outflow cone has a significant component in the line-of-sight and we are looking into the outflow cone (see Fig. 20), this apparent inconsistency may arise from the presence of regions appearing along the same line-of-sight, but which are physically displaced.

In summary, we follow Greenhill et al. (2004b) in that the position angle of the axis of the cone of the outflow in region $\mathrm{B}$ is $\sim 240^{\circ} \pm 10^{\circ}$. We suggest however that the flow, rather than lying in the plane of the sky, lies at an angle of $\sim 45^{\circ}$ to this plane.

A schematic diagram of the region, not to scale, may be found in Fig. 20. This shows the region as if observed from rightangles to the line-of-sight, from a position in Peak 2, looking west. Starting from source I at the base of the diagram, first $\mathrm{H}_{2} \mathrm{O}$ and then $\mathrm{SiO}$ masing regions, overlapping in the line-of-sight, are found in the dense gas close to source I. Moving further from the star, the outflow breaks through the dense gas and dust surrounding source $\mathrm{I}$, generating $\mathrm{H}_{2}$ emission features such as that shown in Fig. 19 and, moving still further, other blue-shifted features shown in Fig. 4 and listed in Table A.3.

We now consider some of the governing parameters of the massive star associated with source I. Greenhill et al. (2004a) found that source I could not be detected at $22 \mu \mathrm{m}$. They concluded that the optical depth at $22 \mu \mathrm{m}$ must be $>300$. Using the relationship between extinction and wavelength given in 
Rosenthal et al. (2000), the NIR optical depth will then be $>570$. This implies that the column density of $\mathrm{H}_{2}$ is $>1.5 \times 10^{25} \mathrm{~cm}^{-2}$. This is comparable with the figure of $\leq 8.5 \times 10^{24} \mathrm{~cm}^{-2}$ estimated in Beuther et al. (2004). Depending on the average gas density around the obscured star, this object must be deeply buried within OMC1. For example if the average density is $10^{9} \mathrm{~cm}^{-3}$, the star is buried to a depth of $\sim 1000 \mathrm{AU}$ and the mass of gas surrounding the star is $9 M_{\odot}$. This is comparable with the estimate of the mass of the star itself of $\leq 10 M_{\odot}$ in Beuther et al. (2004) or the figure of 10-20 $M_{\odot}$ given in Shuping et al. (2004). These masses are consistent with a luminosity of a few thousand to $\sim 10^{4} L_{\odot}$, consistent also with Menten \& Reid (1995). The most recent estimate places the luminosity of source I at $\sim 5 \times 10^{3} L_{\odot}$ (Beuther et al. 2006, 2004). The above mass is also consistent with the calculations of the mass-flow rate for the outflow in Sect. 6.1 which suggested that the driving force was a massive star with a luminosity of $\sim 10^{4} L_{\odot}$.

\subsection{Source $n$ as the origin of the region B outflow}

Source n (Menten \& Reid 1995) is another major power source in the region, in addition to source I and BN. Located a few arcseconds SW of source I (see Fig. 18), source n is clearly another candidate for the origin of the region B outflow. Since source I and n lie so close (Menten \& Reid 1995), geometrical arguments for source I and $\mathrm{n}$ are equally valid. Menten \& Reid (1995) find that the radio emission from source $n$ is elongated in a NE-SW direction, that is, along the region B outflow axis. A disk has recently been observed in the IR around source $\mathrm{n}$ (Greenhill et al. 2004a; Shuping et al. 2004) and this is oriented at right angles to the elongation of source $n$ in the radio. According to Shuping et al. (2004) the disk around source $n$ is inclined $\lesssim 40^{\circ}$ with respect to the plane of the sky, consistent with the inclination of the outflow axis described in the discussion for source I. Furthermore, in the $\mathrm{H}_{2} \mathrm{O}$ maser observations of the outflow in region B (Genzel et al. 1981), the centre of expansion of the masers is found to be close to both source $n$ and source I, with the positional data favouring source n (see Fig. 6 of Genzel et al. 1981) with source $n$ lying within the $1 \sigma$ uncertainties, and source I within $2 \sigma$.

One aspect militating against source $n$ as the source of the region B outflow is that the mass flow rate calculated in Sect. 6.1 is somewhat large for a star with the luminosity of source $n$ ( $\sim 2000 L_{\odot}$, Greenhill et al. 2004a) and source I is the more powerful source.

In conclusion the region B outflow may be powered by either source I or n, perhaps favouring source I on the basis of its greater power output. Source I may however also be the origin of the major NW-SE outflow generating the fingers or this may be powered by a close encounter between source I, source $n$ and BN as suggested by Bally \& Zinnecker (2005). These issues still remain to be resolved.

\section{Conclusions}

The major conclusions of this study using GriF data for the central region of Orion OMC1 may be summarised as follows.

(i) 193 distinct bright $\mathrm{H}_{2}$ emission features in the $v=1-0 \mathrm{~S}(1)$ line have been identified. $67 \%$ of these show clear radial velocity structure with well defined features of greater than $5 \mathrm{~km} \mathrm{~s}^{-1}$, indicating that the emission is generated by shocks in the medium. (ii) Weak radial velocity features (WRVs) with radial velocities $<5 \mathrm{~km} \mathrm{~s}^{-1}$ are strongly concentrated in Peak 1 , and a band of such structures has been identified in a NE-SW direction across Peak 1. In this band, $2 / 3$ of the structures show a broken-up morphology, not seen elsewhere. Possible explanations for this band are discussed.

(iii) Excluding the WRVs, heavily over-represented in Peak 1, but making only a small contribution in Peak 2, there are equal numbers of flows in Peaks 1 and 2. This suggests a greater concentration of activity in Peak 2 which is less than half the size of Peak 1. Flows are found to be grouped much more tightly in Peak 2 than in Peak 1. Blue shifted flows are somewhat favoured over red-shifted, presumably due to dust obscuration. No velocities exceed $35-40 \mathrm{~km} \mathrm{~s}^{-1}$, consistent with the upper limit for the propagation of C-type shocks in dense media.

(iv) In a distinct zone, region B, lying south of Peak 1 and west of Peak 2, all flows are blue-shifted, with an average velocity of $-18 \pm 8 \mathrm{~km} \mathrm{~s}^{-1}$. This is shown to be the infrared counterpart to an outflow previously only identified in radio data. The two possible origins are source I or source n, identified on the basis of the striking positional association, flow speeds and the geometrical relationship between the radio and IR outflows.

(v) Spatial correlation between maximum brightness of $\mathrm{H}_{2}$ emission and maximum velocity reveals deviations typically of $\leq 0.6^{\prime \prime}$. These deviations are be used to estimate the orientation of flows, or shocks, in the plane of the sky - but not their absolute direction, that is, whether they are travelling north or south, say. Orientations are found to be random in Peak 2 and with some clear preference for a SENW direction in Peak 1, but show a strong preference for a $\mathrm{NE}-\mathrm{SW}$ orientation in region $\mathrm{B}$.

(vi) A comparison has been made between the positions of the observed shocks and protostellar candidates in the area using $J, H, K, L$ and X-ray data. We find only a few cases where we can clearly associate shocked gas and protostellar candidates. We conclude that most of the observed bright $\mathrm{H}_{2}$ emission is due to the energy input from the massive stars in the core of $\mathrm{OMC1}$, either directly from the outflow or from supersonic turbulence stirred up by outflows, with a minor contribution from in situ low mass star formation.

(vii) In one position, around a position $0^{\prime \prime} \mathrm{E}, 6^{\prime \prime} \mathrm{N}$ of TCC0016, we identify a region with a high concentration of shock features within an area only a few thousand $\mathrm{AU}$ in diameter which also has several protostellar candidates in and around it. This is a good candidate for a site where multiple low mass star formation is ongoing.

(viii) There is a remarkable feature in the $\mathrm{H}_{2}$ emission data with strong velocity components in region B, shown in Fig. 19. On the basis of a VLT image, this is suggestive of a region in which a flow has burst through the material of OMC1 from behind, sending shock waves through the medium. The axis of the outflow observed in the present work and in extensive radio data, passes through this shock feature.

(ix) There remain several extraordinary objects in the field whose nature we do not comprehend. For example there is a structure, $3^{\prime \prime} \times 3^{\prime \prime}$, Table A.2, object 2-32 and 2-34 in Peak 2, which is the only clump of emission in the entire field with $v_{\mathrm{lsr}}$ consistently around $>40 \mathrm{~km} \mathrm{~s}^{-1}$ (shown in Fig. 4 of Gustafsson et al. 2006b). The origin of such an energetic object is unknown. 
In conclusion, the GriF data with its associated high spatial and velocity resolution provide information which opens a new window on $\mathrm{OMCl}$ and presents a new perspective on the starforming activity for both massive and low mass stars. Data have very recently been obtained for OMC1 using the VLT with adaptive optics and Fabry-Perot interferometry in the $v=1-0 \mathrm{~S}(1)$, $\mathrm{S}(0)$ and the $v=2-1 \mathrm{~S}(1)$ lines. Shock structures seen in these 3 lines will constrain the physical conditions strongly in star forming zones in OMC1. The chief lack at present is of shock models which contain both detailed chemistry, essential for Ctype shocks, and also the geometry, for example of bow shocks. Models are under development and these will be necessary to settle such basic questions as the relative physical disposition of the location of the maximum brightness and maximum velocity in $\mathrm{H}_{2}$ emission features.

Acknowledgements. H.D.N., M.G. and D.F. would like to acknowledge the support of the Aarhus Centre for Atomic Physics (ACAP), funded by the Danish Basic Research Foundation. H.D.N. and M.G. would also like to acknowledge financial support from the Instrument Centre for Danish Astrophysics (IDA), funded by the Danish National Science Committee (SNF, now FNU). J.L.L. would like to acknowledge the support of the PCMI National Program, funded by the French Centre National de la Recherche Scientifique (CNRS). We also wish to thank the Directors and Staff of CFHT and of the VLT for making possible observations reported in this paper and for the assistance rendered by $\mathrm{E}$. Le Coarer (Observatoire de Grenoble) in obtaining GriF data. We also wish to thank G. Pineau des Forêts and Lars E. Kristensen for valuable discussions about the nature of shocks. Our thanks are also due to the referee for many helpful comments, and to whom we owe, among other items, the suggestion that we include details of protostellar candidates and the model that the outflow from IRc9 may be the source of some of the anomalous objects found in our field.

\section{References}

Allen, D. A., \& Burton, M. G. 1993, Nature, 363, 54

Axon, D. J., \& Taylor, K. 1984, MNRAS, 207, 241

Bally, J., \& Zinnecker, H. 2005, AJ, 129, 2281

Bally, J., O'Dell, C. R., \& McCaughrean, M. J. 2000, AJ, 119, 2919

Bate, M. R., Bonnell, I. A., \& Bromm, V. 2003, MNRAS, 339, 577

Beckwith, S., Persson, S. E., Neugebauer, G., \& Becklin, E. E. 1978, ApJ, 223, 464

Beuther, H., Zhang, Q., Greenhill, L. J., et al. 2004, ApJ, 616, L31

Beuther, H., Zhang, Q., Reid, M. J., et al. 2006, ApJ, 636, 323

Blondin, J. M., Konigl, A., \& Fryxell, B. A. 1989, ApJ, 337, L37

Bragg, S. L., Smith, W. H., \& Brault, J. W. 1982, ApJ, 263, 999

Burton, M. G. 1997, in IAU Coll. 163: Accretion Phenomena and Related Outflows, ASP Conf. Ser. 121, 571

Chen, H., Bally, J., O’Dell, C. R., et al. 1998, ApJ, 492, L173

Chrysostomou, A., Burton, M. G., Axon, D. J., et al. 1997, MNRAS, 289, 605

Chrysostomou, A., Gledhill, T. M., Ménard, F., et al. 2000, MNRAS, 312, 103

Clénet, Y., Le Coarer, E., Joncas, G., et al. 2002, PASP, 114, 563

Davis, C. J., Ray, T. P., Desroches, L., \& Aspin, C. 2001, MNRAS, 326, 524

Delgado-Donate, E. J., Clarke, C. J., Bate, M. R., \& Hodgkin, S. T. 2004, MNRAS, 351, 617

Doel, R. C., Gray, M. D., Humphreys, E. M. L., Braithwaite, M. F., \& Field, D. 1995, A\&A, 302, 797

Doi, T., O'Dell, C. R., \& Hartigan, P. 2002, AJ, 124, 445

Dougados, C., Lena, P., Ridgway, S. T., Christou, J. C., \& Probst, R. G. 1993, ApJ, 406, 112

Feigelson, E. D., Broos, P., Gaffney, J. A., et al. 2002, ApJ, 574, 258

Feigelson, E. D., Gaffney, J. A., Garmire, G., Hillenbrand, L. A., \& Townsley, L. 2003, ApJ, 584, 911

Ferland, G. J. 2001, PASP, 113, 41
Field, D., Gerin, M., Leach, S., et al. 1994, A\&A, 286, 909

Flower, D. R., Le Bourlot, J., Pineau des Forêts, G., \& Cabrit, S. 2003, MNRAS, 341,70

Garmire, G., Feigelson, E. D., Broos, P., et al. 2000, AJ, 120, 1426

Genzel, R., \& Stutzki, J. 1989, ARA\&A, 27, 41

Genzel, R., Reid, M. J., Moran, J. M., \& Downes, D. 1981, ApJ, 244, 884

Getman, K. V., Flaccomio, E., Broos, P. S., et al. 2005, ApJS, 160, 319

Gezari, D. Y., Backman, D. E., \& Werner, M. W. 1998, ApJ, 509, 283

Gómez, L., Rodríguez, L. F., Loinard, L., et al. 2005, ApJ, 635, 1166

Greenhill, L. J., Gwinn, C. R., Schwartz, C., Moran, J. M., \& Diamond, P. J. 1998, Nature, 396, 650

Greenhill, L. J., Gezari, D. Y., Danchi, W. C., et al. 2004a, ApJ, 605, L57

Greenhill, L. J., Reid, M. J., Chandler, C. J., Diamond, P. J., \& Elitzur, M. 2004b, in IAU Symp., ed. M. Burton, R. Jayawardhana, \& T. Bourke, 155

Grosso, N., Feigelson, E. D., Getman, K. V., et al. 2005, ApJS, 160, 530

Gustafsson, M., Kristensen, L. E., Clénet, Y., et al. 2003, A\&A, 411, 437 (G2003)

Gustafsson, M., Field, D., Lemaire, J. L., \& Pijpers, F. 2006a, A\&A, 445, 601

Gustafsson, M., Brandenburg, A., Lemaire, J. L., \& Field, D. 2006b, A\&A, 454, 815

Henney, W. J., \& O’Dell, C. R. 1999, AJ, 118, 2350

Hillenbrand, L. A. 1997, AJ, 113, 1733

Kaifu, N., Usuda, T., Hayashi, S. S., et al. 2000, PASJ, 52, 1

Kenyon, S. J., \& Hartmann, L. 1995, ApJS, 101, 117

Koyama, K., Hamaguchi, K., Ueno, S., Kobayashi, N., \& Feigelson, E. D. 1996, PASJ, 48, L87

Kristensen, L. E., Gustafsson, M., Field, D., et al. 2003, A\&A, 412, 727

Kristensen, L. E., Ravkilde, T. L., Field, D., Lemaire, J. L., \& Pineau des Forêts, G. 2006, A\&A, submitted

Kwan, J., \& Scoville, N. 1976, ApJ, 210, L39

Lacombe, F., Gendron, E., Rouan, D., et al. 2004, A\&A, 417, L5

Lada, C. J., Muench, A. A., Haisch, K. E., et al. 2000, AJ, 120, 3162

Lada, C. J., Muench, A. A., Lada, E. A., \& Alves, J. F. 2004, AJ, 128, 1254

Larson, R. B. 2003, Reports of Progress in Physics, 66, 1651

Le Bourlot, J., Pineau des Forêts, G., Flower, D. R., \& Cabrit, S. 2002, MNRAS, 332,985

Lee, J.-K., \& Burton, M. G. 2000, MNRAS, 315, 11

Lim, A. J., Rawling, J. M. C., \& Williams, D. A. 2001, A\&A, 376, 336

McCaughrean, M. J., \& Mac Low, M. 1997, AJ, 113, 391

Menten, K. M., \& Reid, M. J. 1995, ApJ, 445, L157

Muench, A. A., Lada, E. A., Lada, C. J., \& Alves, J. 2002, ApJ, 573, 366

O'Dell, C. R. 2001, ARA\&A, 39, 99

O'Dell, C. R., \& Doi, T. 2003, AJ, 125, 277

O'Dell, C. R., Hartigan, P., Lane, W. M., et al. 1997, AJ, 114, 730

Pudritz, R. E. 2002, Science, 295, 68

Rigaut, F., Salmon, D., Arsenault, R., et al. 1998, PASP, 110, 152

Rodríguez, L. F., Poveda, A., Lizano, S., \& Allen, C. 2005, ApJ, 627, L65

Rosenthal, D., Bertoldi, F., \& Drapatz, S. 2000, A\&A, 356, 705

Salas, L., Rosado, M., Cruz-González, I., et al. 1999, ApJ, 511, 822

Schild, H., Miller, S., \& Tennyson, J. 1997, A\&A, 318, 608

Schultz, A. S. B., Colgan, S. W. J., Erickson, E. F., et al. 1999, ApJ, 511, 282

Shepherd, D. 2005, in IAU Symp. 227, 237

Shuping, R. Y., Morris, M., \& Bally, J. 2004, AJ, 128, 363

Smith, N., \& Bally, J. 2005, ApJ, 622, L65

Störzer, H., \& Hollenbach, D. 1999, ApJ, 515, 669

Stolovy, S. R., Burton, M. G., Erickson, E. F., et al. 1998, ApJ, 492, L151

Stone, J. M., Xu, J., \& Mundy, L. G. 1995, Nature, 377, 315

Tan, J. C. 2004, in Star Formation in the Interstellar Medium: In Honor of David Hollenbach, ASP Conf. Ser. 323, 249

Vannier, L., Lemaire, J. L., Field, D., et al. 2001, A\&A, 366, 651

Wen, Z., \& O’Dell, C. R. 1995, ApJ, 438, 784

Wilgenbus, D., Cabrit, S., Pineau des Forêts, G., \& Flower, D. R. 2000, A\&A, 356,1010

Wright, M., Sandell, G., Wilner, D. J., \& Plambeck, R. L. 1992, ApJ, 393, 225

Wright, M. C. H., Plambeck, R. L., \& Wilner, D. J. 1996, ApJ, 469, 216

Yates, J. A., Field, D., \& Gray, M. D. 1997, MNRAS, 285, 303

Zuckerman, B., Kuiper, T. B. H., \& Rodriguez Kuiper, E. N. 1976, ApJ, 209, L137 
H. D. Nissen et al.: Shocks in Orion, Online Material $p 1$

\section{Online Material}




\section{Appendix A: Tables}

Table A.1. Features identified in OMC1 in Peak 1. Data are sorted by the east-coordinate of the maximum in brightness. Column 1 labels each feature. A "†"-symbol marks the "bipolar pairs" mentioned in Sect. 5.2. Columns 2-5 show the position in the plane of the sky of the maxima in brightness and velocity for each of the features. Positions are measured in arcseconds east and north of the star TCC0016 $\left(05^{\mathrm{h}} 35^{\mathrm{m}} 14.91\right.$, $-05^{\circ} 22^{\prime} 39^{\prime} 31$ (J2000)). Where no coordinates are given for a maximum in velocity, no well-defined peak in the velocity was found nearby. Column 6 shows the velocity of the gas surrounding each feature expressed as $v_{\mathrm{lsr}}$. Column 7 shows the maximum velocity of the flow relative to this local background velocity (see Sect. 3.1). Column 8 contains the maximum brightness of each feature per 400 s exposure. Column 9 shows the position angle for each flow, measured east of north (i.e. counter-clockwise), see Sect. 5.2. A dash indicates that the object is a weak radial velocity region (WRV) and "no p.a." indicates a displacement between maximum velocity and brightness of $<0.25^{\prime \prime}$. A star $\left(^{\star}\right)$ marks flows where the morphology of the emission clearly indicates the absolute direction of the flow (see Sect. 5.1): these are only found in Table A.3. Column 10 gives the displacement in the plane of the sky between the observed maxima in brightness and velocity for each feature. A summary of the data can be seen in Fig. 4.

Flows in Peak 1

\begin{tabular}{|c|c|c|c|c|c|c|c|c|c|}
\hline \multirow{3}{*}{ No. } & \multicolumn{4}{|c|}{ Coordinates of maxima } & \multirow{3}{*}{$\begin{array}{c}\begin{array}{c}\text { Velocity of } \\
\text { surroundings }\end{array} \\
\mathrm{km} \mathrm{s}^{-1}\end{array}$} & \multirow{3}{*}{$\begin{array}{c}\begin{array}{c}\text { Flow } \\
\text { velocity }\end{array} \\
\mathrm{km} \mathrm{s}^{-1}\end{array}$} & \multirow{3}{*}{$\begin{array}{c}\begin{array}{c}\text { Maximum } \\
\text { brightness }\end{array} \\
\text { counts }\end{array}$} & \multirow{3}{*}{$\begin{array}{c}\begin{array}{c}\text { Position } \\
\text { angle }\end{array} \\
\text { degrees }\end{array}$} & \multirow{3}{*}{$\begin{array}{c}\begin{array}{c}\text { Displace- } \\
\text { ment }\end{array} \\
\operatorname{arcsec}\end{array}$} \\
\hline & \multicolumn{2}{|c|}{ Brightness } & \multicolumn{2}{|c|}{ Velocity } & & & & & \\
\hline & $\mathrm{E}$ & $\mathrm{N}$ & E & $\mathrm{N}$ & & & & & \\
\hline $1-1$ & -3.61 & 43.58 & - & - & 17 & - & 204 & - & - \\
\hline $1-2$ & -7.14 & 43.68 & -7.11 & 43.12 & 16 & -6 & 207 & 356 & 0.6 \\
\hline $1-3$ & -7.77 & 22.61 & -7.60 & 22.54 & 8 & -14 & 421 & no p.a. & 0.2 \\
\hline $1-4$ & -8.51 & 46.87 & -8.47 & 47.39 & 20 & 9 & 398 & 184 & 0.5 \\
\hline $1-5$ & -9.48 & 47.28 & - & - & 20 & - & 368 & - & - \\
\hline $1-6$ & -9.52 & 27.09 & - & - & 8 & - & 302 & - & - \\
\hline $1-7$ & -9.90 & 44.94 & - & - & 21 & - & 302 & - & - \\
\hline $1-8$ & -10.22 & 42.46 & -10.05 & 41.90 & 21 & 7 & 264 & 343 & 0.6 \\
\hline $1-9$ & -10.26 & 46.38 & - & - & 20 & - & 366 & - & - \\
\hline $1-10$ & -12.18 & 29.89 & -13.06 & 30.73 & 13 & -16 & 227 & 134 & 1.2 \\
\hline $1-11$ & -12.36 & 36.85 & - & - & 16 & - & 224 & - & - \\
\hline $1-12$ & -12.39 & 28.59 & -11.94 & 28.77 & 13 & 19 & 196 & 249 & 0.5 \\
\hline $1-13$ & -13.16 & 41.16 & - & - & 17 & - & 290 & - & - \\
\hline $1-14$ & -13.61 & 29.05 & - & - & 13 & - & 229 & - & - \\
\hline $1-15$ & -13.86 & 22.61 & -13.48 & 22.43 & 6 & -28 & 187 & 294 & 0.4 \\
\hline $1-16$ & -14.18 & 40.95 & - & - & 18 & - & 328 & - & - \\
\hline $1-17$ & -14.91 & 46.72 & - & - & 22 & - & 259 & - & - \\
\hline $1-18$ & -15.19 & 42.98 & -15.54 & 42.84 & 14 & -9 & 412 & 68 & 0.4 \\
\hline $1-19$ & -15.30 & 38.53 & - & - & 18 & - & 323 & - & - \\
\hline $1-20$ & -15.75 & 32.80 & -16.27 & 32.72 & 6 & -7 & 308 & 82 & 0.5 \\
\hline $1-21$ & -15.86 & 30.80 & -15.54 & 29.47 & 10 & 13 & 324 & 347 & 1.4 \\
\hline $1-22$ & -16.49 & 37.66 & - & - & 14 & - & 271 & - & - \\
\hline $1-23$ & -17.50 & 43.82 & - & - & 12 & - & 426 & - & - \\
\hline $1-24$ & -17.67 & 21.25 & -17.53 & 21.52 & 11 & -10 & 236 & 207 & 0.3 \\
\hline $1-25$ & -17.75 & 36.51 & - & - & 11 & - & 410 & - & - \\
\hline $1-26$ & -18.48 & 35.49 & -18.41 & 34.76 & 9 & -9 & 543 & 355 & 0.7 \\
\hline $1-27$ & -18.51 & 32.72 & - & - & 8 & - & 445 & - & - \\
\hline $1-28$ & -18.59 & 23.34 & -17.64 & 23.76 & 10 & -29 & 227 & 246 & 1.0 \\
\hline $1-29$ & -18.69 & 42.70 & - & - & 14 & - & 409 & - & - \\
\hline $1-30$ & -18.76 & 30.38 & -18.83 & 29.75 & 4 & -8 & 362 & 6 & 0.6 \\
\hline $1-31$ & -19.25 & 21.21 & - & - & 12 & - & 304 & - & - \\
\hline $1-32$ & -19.99 & 25.93 & - & - & 9 & - & 257 & - & - \\
\hline $1-33$ & -20.37 & 28.67 & -20.12 & 28.45 & 8 & -40 & 316 & 311 & 0.3 \\
\hline $1-34$ & -20.58 & 35.98 & -20.26 & 35.00 & 13 & 10 & 483 & 342 & 1.0 \\
\hline $1-35$ & -20.61 & 42.24 & - & - & 16 & - & 286 & - & - \\
\hline $1-36$ & -20.68 & 30.14 & -20.97 & 29.30 & 8 & -17 & 536 & 18 & 0.9 \\
\hline $1-37$ & -21.00 & 32.55 & - & - & 12 & - & 439 & - & - \\
\hline $1-38$ & -21.21 & 33.99 & -21.14 & 33.95 & 12 & -8 & 439 & no p.a. & 0.1 \\
\hline $1-39$ & -21.49 & 37.28 & -21.07 & 37.45 & 14 & -9 & 480 & 247 & 0.5 \\
\hline
\end{tabular}


H. D. Nissen et al.: Shocks in Orion, Online Material p 3

Table A.1. continued.

Flows in Peak 1

\begin{tabular}{|c|c|c|c|c|c|c|c|c|c|}
\hline \multirow{3}{*}{ No. } & \multicolumn{4}{|c|}{ Coordinates of maxima } & \multirow{3}{*}{$\begin{array}{c}\begin{array}{c}\text { Velocity of } \\
\text { surroundings }\end{array} \\
\mathrm{km} \mathrm{s}^{-1}\end{array}$} & \multirow{3}{*}{$\begin{array}{c}\text { Flow } \\
\text { velocity }\end{array}$} & \multirow{3}{*}{$\begin{array}{c}\begin{array}{c}\text { Maximum } \\
\text { brightness }\end{array} \\
\text { counts }\end{array}$} & \multirow{3}{*}{$\begin{array}{c}\begin{array}{c}\text { Position } \\
\text { angle }\end{array} \\
\text { degrees }\end{array}$} & \multirow{3}{*}{$\begin{array}{c}\begin{array}{c}\text { Displace- } \\
\text { ment }\end{array} \\
\operatorname{arcsec}\end{array}$} \\
\hline & \multicolumn{2}{|c|}{ Brightness } & \multicolumn{2}{|c|}{ Velocity } & & & & & \\
\hline & $\mathrm{E}$ & $\mathrm{N}$ & $\mathrm{E}$ & $\mathrm{N}$ & & & & & \\
\hline $1-40$ & -21.84 & 38.64 & -21.84 & 39.87 & 15 & -7 & 401 & 180 & 1.2 \\
\hline $1-41$ & -21.88 & 34.51 & - & - & 12 & - & 416 & - & - \\
\hline $1-42$ & -22.01 & 21.74 & - & - & 7 & - & 223 & - & - \\
\hline $1-43$ & -22.23 & 24.29 & -21.88 & 24.01 & 11 & -9 & 357 & 309 & 0.4 \\
\hline $1-44$ & -22.43 & 30.24 & - & - & 10 & - & 389 & - & - \\
\hline $1-45$ & -22.50 & 18.48 & - & - & 7 & - & 471 & - & - \\
\hline $1-46$ & -22.61 & 19.92 & - & - & 6 & - & 295 & - & - \\
\hline $1-47$ & -23.80 & 35.21 & -23.91 & 35.14 & 12 & 15 & 332 & no p.a. & 0.1 \\
\hline $1-48$ & -24.11 & 37.56 & -23.94 & 36.65 & 13 & -8 & 329 & 349 & 0.9 \\
\hline $1-49$ & -24.33 & 19.67 & -23.98 & 19.57 & 10 & -14 & 413 & 287 & 0.4 \\
\hline $1-50$ & -24.57 & 21.03 & -24.33 & 20.79 & 12 & 13 & 271 & 315 & 0.3 \\
\hline $1-51$ & -24.82 & 28.21 & - & - & 16 & - & 255 & - & - \\
\hline $1-52$ & -25.48 & 29.92 & -25.55 & 29.19 & 14 & 9 & 282 & 5 & 0.7 \\
\hline $1-53$ & -25.66 & 18.20 & - & - & 8 & - & 278 & - & - \\
\hline $1-54$ & -26.04 & 35.53 & -25.76 & 35.35 & 10 & -31 & 349 & 302 & 0.3 \\
\hline $1-55$ & -26.53 & 24.40 & - & - & 17 & - & 301 & - & - \\
\hline $1-56$ & -26.60 & 20.79 & -26.28 & 20.86 & 8 & -5 & 270 & 257 & 0.3 \\
\hline $1-57$ & -26.67 & 32.13 & - & - & 14 & - & 378 & - & - \\
\hline $1-58$ & -27.34 & 33.32 & -26.74 & 33.39 & 12 & -20 & 394 & 263 & 0.6 \\
\hline $1-59$ & -27.55 & 20.06 & - & - & 12 & - & 334 & - & - \\
\hline $1-60$ & -28.17 & 34.33 & -28.49 & 33.25 & 12 & 19 & 498 & 16 & 1.1 \\
\hline $1-61$ & -29.01 & 21.52 & -28.49 & 21.49 & 8 & -32 & 293 & 274 & 0.5 \\
\hline $1-62$ & -29.68 & 38.92 & -29.51 & 38.81 & 19 & -8 & 296 & no p.a. & 0.2 \\
\hline $1-63$ & -29.68 & 23.66 & - & - & 10 & - & 260 & - & - \\
\hline $1-64$ & -30.73 & 25.31 & -31.11 & 25.66 & 15 & 8 & 257 & 132 & 0.5 \\
\hline $1-65$ & -30.84 & 22.50 & - & - & 11 & - & 276 & - & - \\
\hline $1-66$ & -31.50 & 20.75 & - & - & 13 & - & 335 & - & - \\
\hline $1-67$ & -31.89 & 37.62 & - & - & 26 & - & 235 & - & - \\
\hline $1-68$ & -31.99 & 31.57 & -31.92 & 31.11 & 17 & -13 & 266 & 351 & 0.5 \\
\hline $1-69$ & -32.24 & 25.90 & - & - & 18 & - & 257 & - & - \\
\hline $1-70$ & -32.69 & 24.75 & - & - & 18 & - & 251 & - & - \\
\hline $1-71$ & -33.39 & 33.74 & - & - & 28 & - & 241 & - & - \\
\hline $1-72$ & -33.78 & 27.34 & -34.79 & 27.23 & 20 & 15 & 268 & 84 & 1.0 \\
\hline $1-73$ & -35.67 & 39.24 & -35.35 & 38.88 & 18 & -11 & 270 & 318 & 0.5 \\
\hline $1-74$ & -35.84 & 35.07 & - & - & 27 & - & 438 & - & - \\
\hline $1-75$ & -36.22 & 28.70 & -36.92 & 28.63 & 23 & -16 & 178 & 84 & 0.7 \\
\hline $1-76$ & -36.65 & 32.97 & -35.74 & 32.65 & 22 & -16 & 228 & 289 & 1.0 \\
\hline $1-77$ & -37.28 & 21.39 & - & - & 18 & - & 235 & - & - \\
\hline $1-78$ & -37.38 & 24.29 & - & - & 22 & - & 236 & - & - \\
\hline $1-79$ & -37.45 & 32.97 & - & - & 24 & - & 263 & - & - \\
\hline $1-80$ & -37.73 & 23.24 & - & - & 19 & - & 237 & - & - \\
\hline $1-81$ & -38.88 & 32.94 & -39.94 & 32.83 & 24 & 7 & 350 & 84 & 1.1 \\
\hline $1-82$ & -39.17 & 25.83 & - & - & 22 & - & 296 & - & - \\
\hline $1-83$ & -40.60 & 22.19 & - & - & 18 & - & 230 & - & - \\
\hline $1-84$ & -40.95 & 24.29 & - & - & 16 & - & 178 & - & - \\
\hline $1-85$ & -44.80 & 28.94 & - & - & 21 & - & 179 & - & - \\
\hline $1-86$ & -45.29 & 35.46 & -45.57 & 36.12 & 24 & 33 & 178 & 157 & 0.7 \\
\hline $1-87$ & -49.17 & 26.67 & - & - & 20 & - & 185 & - & - \\
\hline
\end{tabular}


H. D. Nissen et al.: Shocks in Orion, Online Material p 4

Table A.2. Flows in Peak 2. For an explanation of each column, see Table A.1.

Flows in Peak 2

\begin{tabular}{|c|c|c|c|c|c|c|c|c|c|}
\hline \multirow{3}{*}{ No. } & \multicolumn{4}{|c|}{ Coordinates of maxima } & \multirow{3}{*}{$\begin{array}{c}\text { Velocity of } \\
\text { surroundings } \\
\mathrm{km} \mathrm{s}^{-1}\end{array}$} & \multirow{3}{*}{$\begin{array}{c}\begin{array}{c}\text { Flow } \\
\text { velocity }\end{array} \\
\mathrm{km} \mathrm{s}^{-1}\end{array}$} & \multirow{3}{*}{$\begin{array}{c}\begin{array}{c}\text { Maximum } \\
\text { brightness }\end{array} \\
\text { counts }\end{array}$} & \multirow{3}{*}{$\begin{array}{c}\begin{array}{c}\text { Position } \\
\text { angle }\end{array} \\
\text { degrees }\end{array}$} & \multirow{3}{*}{$\begin{array}{c}\begin{array}{c}\text { Displace- } \\
\text { ment }\end{array} \\
\text { arcsec }\end{array}$} \\
\hline & \multicolumn{2}{|c|}{ Brightness } & \multicolumn{2}{|c|}{ Velocity } & & & & & \\
\hline & $\mathrm{E}$ & $\mathrm{N}$ & $\mathrm{E}$ & $\mathrm{N}$ & & & & & \\
\hline $2-1$ & 19.39 & -6.51 & 19.25 & -6.62 & 20 & 14 & 261 & no p.a. & 0.2 \\
\hline $2-2$ & 19.00 & 9.45 & 19.42 & 9.41 & 34 & 9 & 233 & 275 & 0.4 \\
\hline $2-3$ & 18.59 & -5.43 & 18.73 & -5.36 & 20 & 9 & 508 & no p.a. & 0.2 \\
\hline $2-4$ & 18.38 & 3.67 & 17.75 & 3.71 & 34 & -12 & 532 & 93 & 0.6 \\
\hline $2-5$ & 17.64 & -0.74 & 17.40 & -1.23 & 24 & -7 & 391 & 27 & 0.5 \\
\hline $2-6$ & 16.76 & 8.72 & 16.84 & 8.72 & 41 & 7 & 407 & no p.a. & 0.1 \\
\hline $2-7$ & 16.34 & 9.73 & - & - & 41 & - & 391 & - & - \\
\hline $2-8$ & 16.20 & -6.47 & 16.10 & -6.51 & 29 & 10 & 357 & no p.a. & 0.1 \\
\hline $2-9^{\dagger} 1$ & 16.17 & -0.49 & 16.66 & -0.67 & 24 & -9 & 331 & 290 & 0.5 \\
\hline $2-10^{\dagger}{ }_{1}$ & 15.82 & -1.61 & 15.47 & -1.12 & 24 & 10 & 860 & 144 & 0.6 \\
\hline $2-11$ & 14.81 & 6.58 & - & - & 34 & - & 287 & - & - \\
\hline $2-12$ & 14.18 & 9.80 & 13.90 & 9.52 & 40 & -8 & 210 & 45 & 0.4 \\
\hline $2-13$ & 13.48 & -1.75 & - & - & 30 & - & 475 & - & - \\
\hline $2-14$ & 13.37 & -0.46 & 13.51 & -0.42 & 30 & 7 & 506 & no p.a. & 0.1 \\
\hline $2-15$ & 12.91 & 8.26 & - & - & 35 & - & 209 & - & - \\
\hline $2-16$ & 12.36 & 0.67 & - & - & 32 & - & 454 & - & - \\
\hline $2-17$ & 12.22 & -2.66 & 12.36 & -3.12 & 25 & -6 & 244 & 343 & 0.5 \\
\hline $2-18$ & 12.15 & 2.28 & - & - & 32 & - & 436 & - & - \\
\hline $2-19$ & 11.65 & 3.95 & 11.62 & 3.88 & 32 & -8 & 404 & no p.a. & 0.1 \\
\hline $2-20$ & 10.81 & 1.23 & 10.57 & 1.64 & 32 & 11 & 456 & 150 & 0.5 \\
\hline $2-21$ & 10.53 & -0.94 & 10.43 & -0.31 & 32 & 8 & 361 & 171 & 0.6 \\
\hline $2-22$ & 10.40 & 8.75 & 10.81 & 8.78 & 34 & -7 & 188 & 265 & 0.4 \\
\hline $2-23$ & 10.11 & -6.51 & - & - & 23 & - & 273 & - & - \\
\hline $2-24$ & 9.66 & 1.19 & 9.84 & 1.01 & 31 & 7 & 349 & no p.a. & 0.2 \\
\hline $2-25$ & 9.56 & 2.17 & 9.38 & 3.01 & 31 & -10 & 341 & 168 & 0.9 \\
\hline $2-26$ & 8.86 & 1.78 & 8.78 & 1.78 & 31 & -12 & 370 & no p.a. & 0.1 \\
\hline $2-27$ & 7.70 & 6.30 & 8.12 & 6.20 & 30 & -7 & 220 & 284 & 0.4 \\
\hline $2-28$ & 7.53 & -3.64 & - & - & 27 & - & 308 & - & - \\
\hline $2-29$ & 7.07 & -1.05 & 7.03 & -1.01 & 30 & -6 & 335 & no p.a. & 0.0 \\
\hline $2-30$ & 6.82 & -4.69 & - & - & 26 & - & 308 & - & - \\
\hline $2-31$ & 6.62 & 6.86 & 6.51 & 6.65 & 26 & -11 & 182 & no p.a. & 0.2 \\
\hline $2-32$ & 6.62 & 2.62 & 6.72 & 2.73 & 30 & 32 & 283 & no p.a. & 0.1 \\
\hline $2-33$ & 6.51 & -3.05 & 6.47 & -3.01 & 28 & -6 & 350 & no p.a. & 0.0 \\
\hline $2-34$ & 6.30 & 1.09 & 6.86 & 1.33 & 30 & 26 & 310 & 246 & 0.6 \\
\hline $2-35$ & 6.30 & -0.56 & 6.30 & -0.42 & 30 & 15 & 498 & no p.a. & 0.1 \\
\hline $2-36$ & 5.95 & 7.18 & 5.99 & 7.24 & 26 & -13 & 194 & no p.a. & 0.1 \\
\hline $2-37$ & 5.25 & -3.33 & 5.14 & -3.05 & 32 & 10 & 530 & 159 & 0.3 \\
\hline $2-38$ & 5.07 & -2.77 & 5.07 & -2.24 & 30 & 9 & 323 & 180 & 0.5 \\
\hline $2-39$ & 4.80 & -8.23 & 4.24 & -7.98 & 20 & -7 & 205 & 114 & 0.6 \\
\hline $2-40$ & 4.62 & 10.85 & 4.41 & 10.95 & 20 & -18 & 531 & no p.a. & 0.2 \\
\hline $2-41$ & 3.82 & -2.38 & 3.64 & -2.56 & 31 & 11 & 209 & no p.a. & 0.2 \\
\hline $2-42$ & 3.12 & 7.66 & 3.01 & 7.45 & 20 & -8 & 305 & no p.a. & 0.2 \\
\hline $2-43$ & 2.62 & 0.94 & 2.66 & 1.09 & 20 & -7 & 232 & no p.a. & 0.1 \\
\hline $2-44$ & 1.92 & 1.54 & 1.68 & 1.58 & 20 & -14 & 227 & no p.a. & 0.2 \\
\hline $2-45^{\dagger_{2}}$ & 1.58 & 5.28 & 1.78 & 4.93 & 18 & 6 & 542 & 329 & 0.4 \\
\hline $2-46$ & 1.37 & 4.03 & 1.92 & 3.67 & 15 & 4 & 377 & 302 & 0.7 \\
\hline $2-47^{\dagger}{ }_{2}$ & 0.74 & 6.05 & 0.77 & 6.41 & 10 & -13 & 372 & 186 & 0.4 \\
\hline $2-48$ & 0.14 & 4.03 & -0.42 & 4.13 & 10 & -8 & 316 & 101 & 0.6 \\
\hline $2-49$ & -0.07 & 8.05 & -0.14 & 8.30 & 12 & -15 & 229 & 164 & 0.3 \\
\hline $2-50$ & -1.05 & 9.80 & - & - & 16 & - & 192 & - & - \\
\hline
\end{tabular}


H. D. Nissen et al.: Shocks in Orion, Online Material p 5

Table A.3. Flows in region B. For an explanation of each column, see Table A.1.

Flows in region B

\begin{tabular}{|c|c|c|c|c|c|c|c|c|c|}
\hline \multirow{3}{*}{ No. } & \multicolumn{4}{|c|}{ Coordinates of maxima } & \multirow{3}{*}{$\begin{array}{c}\begin{array}{c}\text { Velocity of } \\
\text { surroundings }\end{array} \\
\mathrm{km} \mathrm{s}^{-1}\end{array}$} & \multirow{3}{*}{$\begin{array}{c}\begin{array}{c}\text { Flow } \\
\text { velocity }\end{array} \\
\mathrm{km} \mathrm{s}^{-1}\end{array}$} & \multirow{3}{*}{$\begin{array}{c}\begin{array}{c}\text { Maximum } \\
\text { brightness }\end{array} \\
\text { counts }\end{array}$} & \multirow{3}{*}{$\begin{array}{c}\begin{array}{c}\text { Position } \\
\text { angle }\end{array} \\
\text { degrees }\end{array}$} & \multirow{3}{*}{$\begin{array}{c}\begin{array}{c}\text { Displace- } \\
\text { ment }\end{array} \\
\operatorname{arcsec}\end{array}$} \\
\hline & \multicolumn{2}{|c|}{ Brightness } & \multicolumn{2}{|c|}{ Velocity } & & & & & \\
\hline & E & $\mathrm{N}$ & E & $\mathrm{N}$ & & & & & \\
\hline B-1 & -2.20 & 5.60 & -2.13 & 5.46 & 4 & -14 & 240 & no p.a. & 0.2 \\
\hline B-2 & -3.26 & -12.43 & -3.61 & -12.36 & 22 & -9 & 174 & 101 & 0.4 \\
\hline B-3 & -3.99 & 6.12 & -3.61 & 6.72 & 5 & -22 & 348 & 213 & 0.7 \\
\hline B-4 & -4.41 & -6.05 & -4.83 & -5.70 & 22 & -8 & 192 & 130 & 0.5 \\
\hline B-5 & -4.66 & 5.70 & -5.57 & 5.64 & 5 & -34 & 432 & 86 & 0.9 \\
\hline B-6 & -4.93 & -8.89 & -5.04 & -8.89 & 22 & -7 & 145 & no p.a. & 0.1 \\
\hline B-7 & -7.77 & 7.80 & -7.95 & 7.84 & 7 & -28 & 208 & no p.a. & 0.2 \\
\hline B-8 & -8.05 & 13.90 & -7.98 & 13.69 & 14 & -24 & 255 & no p.a. & 0.2 \\
\hline B-9 & -8.05 & 7.66 & -7.24 & 7.32 & 7 & -20 & 199 & 293 & 0.9 \\
\hline B-10 & -8.19 & 7.32 & -7.53 & 7.00 & 7 & -24 & 198 & 295 & 0.7 \\
\hline B-11 & -8.36 & 10.29 & -8.26 & 10.36 & 12 & -12 & 175 & no p.a. & 0.1 \\
\hline B-12 & -8.68 & 14.77 & -8.78 & 14.56 & 14 & -21 & 365 & no p.a. & 0.2 \\
\hline B-13 & -8.93 & 13.61 & -9.17 & 13.12 & 14 & -10 & 343 & 27 & 0.5 \\
\hline B-14 & -9.69 & -8.75 & -9.80 & -8.72 & 27 & -13 & 181 & no p.a. & 0.1 \\
\hline B-15 & -9.90 & -8.19 & -9.98 & -8.05 & 27 & -14 & 182 & no p.a. & 0.2 \\
\hline B-16 & -10.64 & -0.49 & -10.71 & -0.07 & 13 & -13 & 261 & 171 & 0.4 \\
\hline B-17 & -10.99 & -16.94 & - & - & 26 & - & 231 & - & - \\
\hline B-18 & -11.80 & 2.13 & -11.94 & 2.38 & 7 & -13 & 362 & 150 & 0.3 \\
\hline B-19 & -11.94 & 1.58 & -11.94 & 1.54 & 7 & -16 & 392 & no p.a. & 0.0 \\
\hline B-20 & -12.32 & 1.50 & -12.49 & 1.58 & 7 & -18 & 368 & no p.a. & 0.2 \\
\hline B-21 & -12.81 & 2.94 & -12.64 & 2.97 & 7 & -14 & 365 & no p.a. & 0.2 \\
\hline B-22 & -13.12 & 1.92 & -13.23 & 2.00 & 7 & -17 & 307 & no p.a. & 0.1 \\
\hline B-23 & -13.69 & -0.52 & -13.30 & 0.07 & 8 & -20 & 531 & $213^{\star}$ & 0.7 \\
\hline B-24 & -13.76 & 11.44 & -13.27 & 11.86 & 3 & -16 & 226 & 229 & 0.6 \\
\hline B-25 & -14.35 & 11.90 & -13.82 & 12.01 & 3 & -23 & 212 & 259 & 0.5 \\
\hline B-26 & -14.73 & -7.56 & -14.45 & -6.89 & 20 & -7 & 178 & $203^{\star}$ & 0.7 \\
\hline B-27 & -15.36 & -11.90 & -15.36 & -11.62 & 10 & -12 & 200 & 180 & 0.3 \\
\hline B-28 & -15.89 & 12.95 & -14.77 & 13.06 & 3 & -20 & 216 & 265 & 1.1 \\
\hline B-29 & -15.99 & 7.28 & -15.82 & 7.00 & 7 & -20 & 163 & 328 & 0.3 \\
\hline B-30 & -16.62 & 13.93 & -16.66 & 13.90 & 3 & -11 & 167 & no p.a. & 0.0 \\
\hline B-31 & -16.73 & 5.99 & - & - & 7 & - & 221 & - & - \\
\hline B-32 & -17.29 & 8.68 & -17.15 & 8.36 & 8 & -20 & 190 & 336 & 0.3 \\
\hline B-33 & -17.57 & 10.05 & -17.57 & 9.98 & 4 & -17 & 400 & no p.a. & 0.1 \\
\hline B-34 & -17.60 & 7.80 & -17.60 & 7.95 & 8 & -20 & 206 & no p.a. & 0.1 \\
\hline B-35 & -17.78 & 0.17 & -17.40 & -0.38 & 10 & -26 & 358 & 325 & 0.7 \\
\hline B-36 & -17.95 & -1.15 & -17.82 & -2.13 & 10 & -37 & 404 & 352 & 1.0 \\
\hline B-37 & -18.48 & 0.00 & -17.85 & 0.38 & 10 & -18 & 363 & $239^{\star}$ & 0.7 \\
\hline B-38 & -18.73 & 2.52 & -18.51 & 3.05 & 15 & -14 & 234 & 202 & 0.6 \\
\hline B-39 & -18.76 & 5.46 & - & - & 10 & - & 239 & - & - \\
\hline B-40 & -18.90 & 7.95 & - & - & 6 & - & 281 & - & - \\
\hline B-41 & -19.15 & 6.65 & -19.08 & 6.72 & 8 & -19 & 301 & no p.a. & 0.1 \\
\hline B-42 & -19.67 & 16.24 & -19.84 & 16.91 & 7 & -19 & 401 & $165^{\star}$ & 0.7 \\
\hline B-43 & -20.44 & -6.76 & -20.23 & -6.51 & 7 & -36 & 291 & $221^{\star}$ & 0.3 \\
\hline B-44 & -22.78 & 7.28 & -22.78 & 7.32 & 10 & -39 & 173 & no p.a. & 0.0 \\
\hline B-45 & -23.10 & 6.05 & -22.54 & 6.12 & 10 & -15 & 208 & 263 & 0.6 \\
\hline B-46 & -23.91 & 6.34 & -23.91 & 6.34 & 10 & -29 & 293 & no p.a. & 0.0 \\
\hline B-47 & -24.75 & 0.88 & -24.85 & 0.98 & 8 & -20 & 256 & no p.a. & 0.1 \\
\hline B-48 & -25.48 & 16.34 & -25.02 & 16.45 & 5 & -14 & 356 & 257 & 0.5 \\
\hline B-49 & -25.83 & 0.35 & -25.66 & 0.46 & 7 & -7 & 188 & no p.a. & 0.2 \\
\hline B-50 & -27.26 & 16.49 & -27.34 & 16.45 & 5 & -15 & 305 & no p.a. & 0.1 \\
\hline B-51 & -27.55 & 11.65 & -26.99 & 12.01 & 12 & -19 & 273 & 238 & 0.7 \\
\hline B-52 & -30.03 & -8.40 & - & - & 18 & - & 379 & - & - \\
\hline B-53 & -30.91 & -10.08 & - & - & 18 & - & 368 & - & - \\
\hline B-54 & -31.26 & 5.95 & -30.94 & 6.02 & 17 & -7 & 314 & 257 & 0.3 \\
\hline B-55 & -33.28 & 7.18 & - & - & 18 & - & 249 & - & - \\
\hline B-56 & -33.92 & 16.17 & -33.35 & 16.10 & 12 & -9 & 202 & 277 & 0.6 \\
\hline
\end{tabular}

